\title{
Episodios de Cambio y Estancamiento en Psicoterapia: Características de la comunicación verbal entre pacientes y terapeutas ${ }^{1}$
}

\author{
Change and Stuck Episodes in Psychotherapy: Characterization of verbal \\ communication between patients and therapists
}

\author{
Olga Fernández \\ Pablo Herrera \\ Mariane Krause \\ Pontificia Universidad Católica de Chile, Chile \\ Janet Carola Pérez \\ Universidad del Desarrollo, Pontificia Universidad Católica de Chile, Chile \\ Nelson Valdés \\ Pontificia Universidad Católica de Chile, Chile \\ Oriana Vilches \\ Universidad de Valparaíso, Chile \\ $\&$ \\ Alemka Tomicic \\ Universidad del Desarrollo, Pontificia Universidad Católica de Chile, Chile
}

(Rec: 30 de diciembre de 2011 / Acep: 20 de mayo de 2012)

\section{Resumen}

Se presentan los resultados de un microanálisis de la conversación psicoterapéutica en Episodios de Cambio y de Estancamiento. Se realizó un análisis comparativo de la comunicación verbal en una muestra de 33 Episodios de Cambio y 34 de Estancamiento, correspondientes a 15 procesos terapéuticos, aplicando el Sistema de Codificación de la Actividad Terapéutica. Este sistema distingue cinco niveles de análisis: Formas Básicas, Intenciones Comunicacionales, Técnicas, Dominio y Referencia. Los resultados muestran que los Episodios de Cambio y Estancamiento se distinguen en cuanto a que, en los primeros, predomina un lenguaje más asertivo por parte del terapeuta y más receptivo en el paciente, estando ambos involucrados en la activa construcción de nuevos significados y el paciente centrado en sí mismo. En los segundos se rigidiza el lenguaje, se exacerban algunas técnicas por parte del terapeuta y se tiende a focalizar menos en el paciente como referente del diálogo terapéutico.

Palabras clave: Proceso terapéutico, cambio, episodios relevantes, acciones comunicacionales.

\begin{abstract}
Results of a microanalysis of the psychotherapeutic conversation in change and stuck episodes are presented. For the comparison of the verbal communication, the Therapeutic Activity Coding System was applied to a sample of 33 change episodes and 34 stuck episodes, corresponding to 15 therapeutic processes. This system includes five levels of analysis: Basic Forms, Communicative Intentions, Techniques, Domain and Reference. Results show differences in change and stuck episodes. The former are characterized by an assertive speech on the therapists' side, and a more receptive language from the patients, with both actors involved in an active construction of new meanings, and the patients referring to him or herself. In contrast, the language in stuck episodes is less flexible, the use of some therapist techniques is exacerbated and the therapeutic dialogue tends to be less focused on the patient as a reference point.
\end{abstract}

Keywords: therapeutic process, change, relevant episodes, communicative actions.

Correspondencia: La correspondencia relativa a este artículo debe ser dirigida a: Olga Fernández, Programa de Doctorado en Psicoterapia, Pontificia Universidad Católica de Chile, Vicuña Mackenna 4860, Macul, Santiago, Chile. E-mail: ofernandezg@uc.cl

1 Los resultados de investigación presentados en este artículo forman parte del Proyecto Fondecyt $\mathrm{N}^{\circ} 1080136$ (Fondo Nacional para el Desarrollo de la Ciencia y la Tecnología, Ministerio de Educación, Gobierno de Chile), institución a la que agradecemos su apoyo. 
Olga Fernández, Pablo Herrera, Mariane Krause, Janet Carola Pérez,

Nelson Valdés, Oriana Vilches \& Alemka Tomicic

\section{Introducción}

El lenguaje, verbal y no verbal, es por excelencia la herramienta psicoterapéutica. En la mayoría de las modalidades terapéuticas, el proceso de cambio se construye a través de la comunicación entre terapeutas y consultantes (Elliott, Slatick \& Urman, 2001; Krause et al., 2007) o incluso, como sostiene la teoría dialógica del self, implica el diálogo entre distintas posiciones del yo (Aristegui et al., 2009; Martínez, 2011). Si bien la investigación en psicoterapia también alberga vertientes que estudian aspectos no verbales de la comunicación (Bänninger-Huber, 1992; Bänninger-Huber \& Widmer, 1999; Tomicic, Martínez, Chacón \& Guzmán, 2011), es el lenguaje hablado el que ha recibido históricamente la mayor atención (Friedlander, 1982). De hecho, utilizar la palabra como instrumento de sanación -la cura por la palabra- (Breuer \& Freud, 1895), es una de las diferencias esenciales de la psicoterapia con otras formas de sanar.

El cambio psicoterapéutico, entendido desde la Teoría del Cambio Subjetivo (Krause, 2005) como un cambio en los patrones subjetivos de interpretación y explicación que llevan al desarrollo de nuevas teorías comprensivas de sí mismo, es construido paso a paso a través de conexiones de significados que se van elaborando en la interacción verbal.

Sin embargo, este proceso de construcción verbal de significados no se supone homogéneo; de hecho, la evidencia empírica que sustenta la existencia de segmentos de la terapia particularmente ligados al cambio -versus otros en que éste no acontece en la misma medida- es abundante y se ha ido acumulando durante más de tres décadas (Bastine, Fiedler \& Kommer, 1989; Elliott, 1984; Gonçalves, Matos \& Santos, 2009; entre otros). El análisis de la comunicación verbal, desde esta perspectiva del estudio de episodios relevantes, ha podido caracterizar las verbalizaciones asociadas al cambio. De hecho, el estudio de momentos significativos permite una mirada microanalítica a las secuencias de interacciones, lo que además de contribuir a comprender la construcción del cambio, entrega aportes a la práctica clínica porque observa el accionar dentro de la sesión de trabajo terapéutico (Elliott, 2010). Los resultados de estudios empíricos han revelado algunas características de estos momentos significativos. Por ejemplo, Reyes et al. (2008) mostraron -desde un análisis de actos de habla- que los momentos de cambio se caracterizaban por verbalizaciones expresadas en tiempo presente, en primera persona y con un contenido autorreferencial; en tanto el estudio de Valdés, Krause y Álamo (2011), que analiza las verbalizaciones a través del Sistema de Codificación de la Actividad Terapéutica (SCAT-1.0; Valdés et al., 2010), mostró el rol complementario de terapeutas y consultantes en la comunicación durante Episodios de Cambio.

Lo que busca aportar el presente estudio es profundizar la caracterización de la comunicación en segmentos relevantes del microproceso terapéutico, analizando comparativamente las verbalizaciones contenidas en dos tipos de episodios contrastantes como lo son los Episodios de Cambio y los Episodios de Estancamiento.

\section{Episodios Relevantes en Psicoterapia}

La investigación en procesos de cambio en psicoterapia ha utilizado ampliamente la aproximación a eventos o momentos significativos durante la terapia. Entre los segmentos significativos, los más estudiados han sido aquellos vinculados con el cambio, y que han recibido diferentes denominaciones, tales como Eventos de Empoderamiento (Timulak \& Elliott, 2003), Momentos Innovativos (Gonçalves et al., 2009; Matos, Santos, Goncalves \& Martins, 2009), Insight (Elliott, 1984) y Momentos de Cambio (Krause et al., 2006), entre otros.

También, aunque menos abundantemente, se han investigado momentos de la terapia que dificultan el cambio o que se oponen a éste, conceptualizados como "momentos difíciles" durante el proceso terapéutico (Herrera, Fernández, Krause, Vilches, Valdés, \& Dagnino, 2009), entre los que se distinguen, dependiendo de los énfasis y del foco de observación: Rupturas (Safran \& Muran, 2000), Refusal (Billow, 2007), Reactancia (Brehm \& Brehm, 1981), Resistencia (Arkowitz, 2002), Impasse (Etchegoyen, 1986), y Estancamiento (Herrera et al., 2009).

En la presente investigación se trabajó con Episodios de Cambio y de Estancamiento. Los Episodios de Cambio (EC) son segmentos de la sesión en los cuales se puede identificar un Momento de Cambio, como evento final. Siguiendo los postulados de la Teoría del Cambio Subjetivo (Krause, 2005), los EC se conceptualizan como segmentos de la terapia que implican transformaciones en la subjetividad del cliente, cuyo contenido es de una complejidad creciente y puede ser rotulado de acuerdo con la Jerarquía de Indicadores de Cambio Genéricos (Krause et al., 2007; véase lista en anexo 1).

Los Episodios de Estancamiento se caracterizan por la detención temporal del proceso de cambio del consultante, debido a la reedición de un patrón disfuncional de éste durante la sesión terapéutica (Herrera et al., 2009). Esta reedición se caracteriza por su persistencia en formas de entendimiento, comportamiento y emociones relacionadas con su problema. Al igual que en los Episodios de Cambio, el foco de la observación está puesto en el cliente, independientemente de la acción u omisión del terapeuta y de sus posibles aciertos o desaciertos. Así como en los Episodios de Cambio se construyen nuevos significados, los de Estancamiento se caracterizan por la falta de construcción de nuevos modos de interpretación o representación. Los contenidos de los Episodios de Estancamiento se etiquetan usando la Lista de Temáticas de Estancamiento (véase lista en anexo 2). 


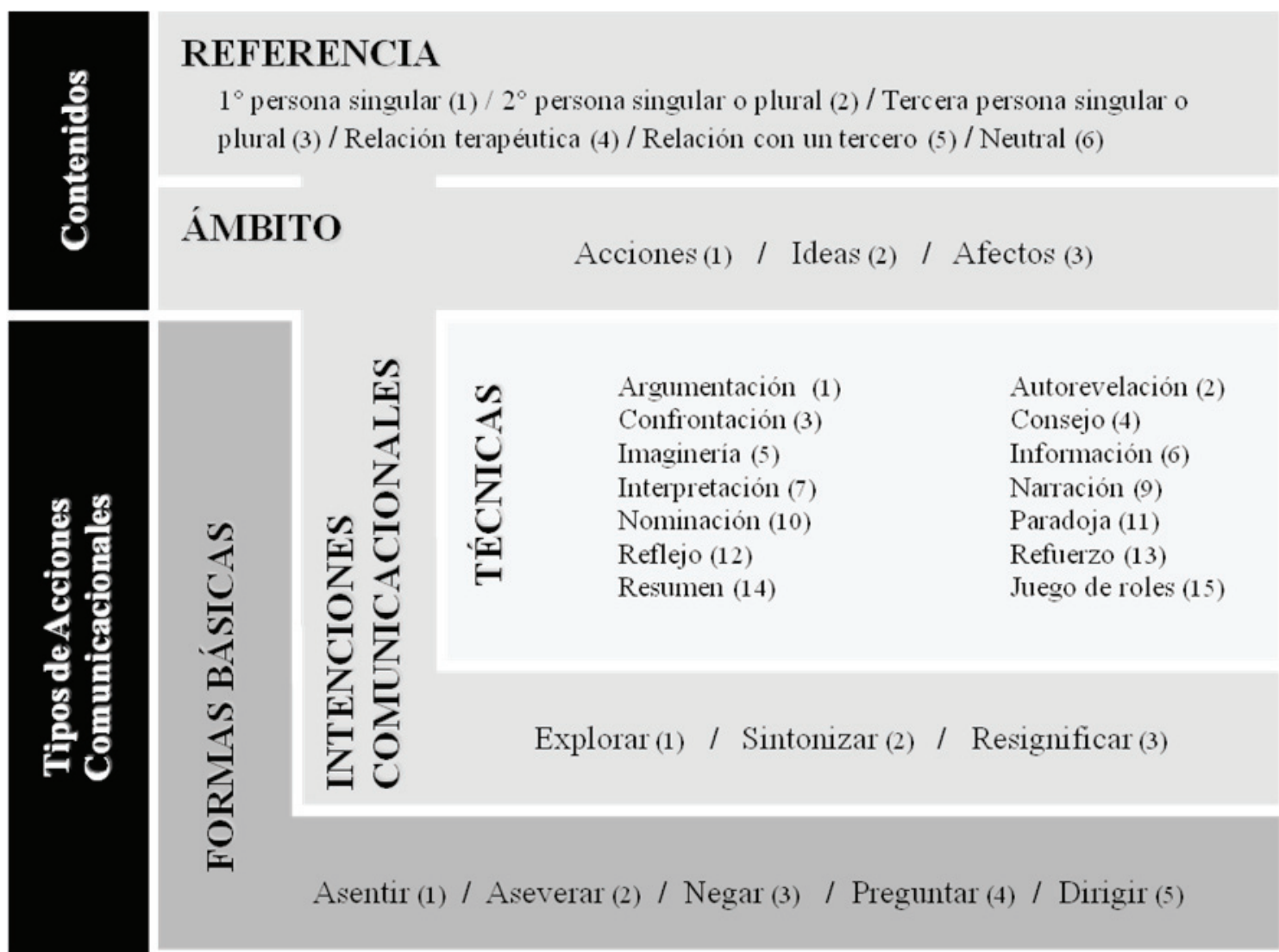

Figura 1. Dimensiones, categorías y códigos del Sistema de Codificación de la Actividad Terapéutica (SCAT-1.0, Valdés et al., 2010b).

Ambos tipos de episodio, cuyos criterios metodológicos de identificación serán desarrollados en la sección método, tienen una duración de alrededor de 3 minutos, y suelen estar compuestos por entre 20 y 50 turnos de palabra, a los que se aplica el análisis comunicacional a través del Sistema de Codificación de la Actividad Terapéutica (SCAT-1.0, Valdés et al., 2010).

\section{Sistema de Codificación de la Actividad Terapéutica (SCAT-1.0)}

El SCAT-1.0 (Valdés et al., 2010), es un sistema de codificación del lenguaje terapéutico "meta-escuela", que permite captar las dimensiones relevantes de las verbalizaciones que componen el diálogo terapéutico, buscando características que son comunes a los distintos enfoques y modalidades de terapia (Valdés et al., 2011), sin centrarse específicamente en problemas psicológicos ni en las intervenciones terapéuticas particulares de algunos enfoques o modalidades. Este sistema de codificación fue construido para responder a la necesidad de conceptualizar e investigar la actividad verbal de pacientes y terapeutas en el contexto de la psicoterapia. Tiene en la base una idea performativa del lenguaje (Searle, 1992, 2002). De acuerdo con este planteamiento conceptual, "las verbalizaciones son llamadas
Acciones Comunicacionales puesto que cumplen con el doble propósito de transportar información (Contenidos) y ejercer influencia sobre el otro y la realidad construida por los hablantes (Acción)" (Valdés et al., 2011, p. 18). Así, este sistema de codificación considera tanto la dimensión de acción como la dimensión de contenido de las Acciones Comunicacionales. Las primeras incluyen Formas Básicas, Intenciones Comunicacionales y Técnicas, en tanto las segundas consideran el Ámbito y la Referencia.

Categorías de la dimensión de Acción. La primera de estas categorías se denomina Forma Básica ${ }^{2} y$ permite clasificar la estructura formal de la verbalización realizada por el hablante, distinguiendo entre los códigos: Asentir, Aseverar, Negar, Preguntar y Dirigir. La Forma Básica Asentir es la Acción Comunicacional realizada por el hablante para admitir como cierto lo dicho por el otro (Ej., "claro", "por supuesto", "puede ser", "mhm"), mientras que la Forma Básica Aseverar es realizada por el hablante para afirmar algo que pretende ser verdad (Ej., "pero igual la echa de menos, esa podría ser otra manera", "lo hizo para comprometerse", "imposible”). La Forma Básica Negar

A lo largo de todo el artículo, las categorías del SCAT-1.0 (Forma Básica, Intención Comunicacional, Técnica, Ámbito y Referencia) aparecerán $\underline{\text { subrayadas, }}$ mientras que sus respectivos códigos aparecerán en cursiva. 
es realizada por el hablante para no admitir como cierto lo dicho por el otro (Ej., "no", "de ninguna manera"), y la Forma Básica Preguntar es la Acción Comunicacional realizada por el hablante para interrogar al otro sobre cierta información (Ej., “¿y de qué cosa se quejaba entonces?”, “¿cómo se sintió en ese momento?"). Finalmente, la Forma Básica Dirigir es realizada para intentar que el otro ejecute una acción cognitiva, emocional o comportamental (Ej., "piense en lo que le acabo de decir", "sostenga la emoción que está sintiendo ahora", "mire a su marido mientras le habla"). La segunda categoría de la dimensión Acción se denomina Intención Comunicacional y permite clasificar el propósito comunicacional expresado durante la verbalización del hablante, vale decir, lo que la persona persigue con su comunicación. Esta categoría permite distinguir entre los códigos: Explorar, Sintonizar y Resignificar. La Intención Comunicacional Explorar es realizada por el hablante con el propósito de: (a) averiguar o entregar contenidos que se desconocen (“¿a qué le teme?", "mi madre tenía entonces 36 años"); (b) clarificar contenidos (" ¿o sea que esas cosas no las cuentas?"); y/o (c) dirigir la atención y los esfuerzos hacia un determinado foco de la conversación ("voy a contarle algo que me pasó el fin de semana"). La Intención Comunicacional Sintonizar es realizada con el propósito de: (a) entender o ser entendido por el otro ("necesito que me entienda lo que intento explicarle", "ahh, lo que me quieres decir es que te cuesta reconocerlo"); (b) armonizar con el otro ("me pongo en tu lugar y para ti debe ser muy complicado todo esto"); y/o (c) darle una retroalimentación al otro ("lo que acabas de decir me molestó"). Finalmente, la Intención Comunicacional Resignificar es la Acción Comunicacional realizada con el propósito de: (a) construir nuevos significados ("usted no se ha puesto a pensar que hay una parte suya que anda por la vida como aplanadora, y otra parte suya que nadie ve. Esa es la parte que sufre"); y/o (b) consolidarlos ("en el fondo fondo, nunca quise dejar de ser niña").

La tercera categoría de la dimensión Acción se denomina Técnica y permite clasificar los recursos metodológicos comunicacionales presentes en la verbalización del hablante, distinguiendo entre los códigos: Argumentación, Autorrevelación, Confrontación, Consejo, Imaginería, Información, Interpretación, Narración, Nominación, Paradoja, Reflejo, Refuerzo, Resumen y Juego de Roles (Role Playing) (Valdés et al., 2010).

Categorías de la dimensión Contenido. La primera categoría de la dimensión contenido se denomina Ámbito y permite clasificar la Acción Comunicacional, considerando para ello si el objeto del trabajo terapéutico es principalmente comportamental (ámbito de las acciones), cognitivo (ámbito de las ideas), o emocional (ámbito de los afectos) (Valdés et al., 2010).

Finalmente, la segunda categoría de la dimensión Contenido se denomina Referencia y permite clasificar la
Acción Comunicacional dependiendo de si está dirigida a si mismo (paciente o terapeuta, formulada en primera persona singular), a otro presente en la sesión (paciente, terapeuta u otros, formulada en segunda persona singular o plural), a un tercero fuera de la sesión (tercera persona singular o plural), a la relación terapéutica, a la relación con un tercero fuera de la sesión, o si se refiere a un sujeto neutral (Valdés et al., 2010).

El Sistema de Codificación de la Actividad Terapéutica (SCAT-1.0, Valdés et al., 2010) ha permitido realizar algunos estudios donde se ha analizado el habla en momentos de cambio, así como también se han podido comparar sus resultados con otros sistemas de análisis de lenguaje en la terapia. Valdés (2010) y Valdés et al. (2011) han analizado el habla en episodios de cambio, aplicando el SCAT-1.0 y el Buscador Lingüístico y Contador de Palabras (LIWC), encontrando diferencias entre el paciente y el terapeuta en Episodios de Cambio. Por ejemplo en la dimensión Acción, se vio que el cliente asiente y niega más, mientras que el terapeuta pregunta más; el cliente comunica más con la Intención Comunicacional de dar información mientras que el terapeuta busca más Sintonizar con el paciente y Resignificar lo que éste relata. Respecto de la dimensión Contenido, se observó que el paciente habla más de si mismo, de terceras personas y de su relación con otros, mientras que el terapeuta habla más sobre el paciente y la relación terapéutica (Valdés, 2010).

Por su parte, Reyes et al. (2008) ha descrito el lenguaje en Episodios de Cambio, en particular en el sub-segmento denominado momento de cambio, caracterizándolo por el uso de la primera persona del presente indicativo por parte del consultante, en un diálogo con su terapeuta a través del que se va construyendo una posición nueva del yo. Un Episodio de Estancamiento, por el contrario, no generaría un contexto de diálogo ni autodiálogo terapéutico alternativo: no hay escuchas, no se afectan el uno al otro, y por lo tanto no puede construirse un nuevo significado (Arístegui et al., 2009).

Estos estudios previos constituyen una aproximación al problema central del presente trabajo, que es la distinción entre Episodios de Cambio y de Estancamiento, pero sólo parcialmente, ya que en un caso el énfasis estuvo puesto en la distinción del lenguaje de pacientes y terapeutas (Valdés, 2010) y en el otro en la distinción general de Episodios de Cambio y Estancamiento, pero sin considerar las cinco categorías de análisis de las Acciones Comunicacionales previamente presentadas. El presente estudio aporta entonces, en forma adicional a los conocimientos existentes, la distinción entre Episodios de Cambio y Estancamiento en todos los niveles de análisis de las Acciones Comunicacionales que aporta el SCAT-1.0, y distinguiendo entre pacientes y terapeutas. 


\section{Método}

En este estudio se utilizó una metodología mixta que contó con una primera fase cualitativa, durante la cual fueron identificados y delimitados todos los episodios que conformaban la muestra total y se codificaron los turnos de palabra contenidos en una muestra de dichos episodios y, una segunda fase, cuantitativa, durante la cual se realizaron análisis estadísticos de las diferentes acciones comunicacionales presentes en los turnos de palabra de Episodios de Cambio versus de Estancamiento.

\section{Muestra}

Se registró el total de sesiones $(\mathrm{N}=294)$ de 15 procesos terapéuticos, con el objeto de seleccionar, delimitar y transcribir todos los Episodios de Cambio y Episodios de Estancamiento contenidos en éstos. Coincidentemente con el objetivo de analizar la comunicación terapéutica en forma transteórica, las terapias incluidas fueron de distintas modalidades (individual, de pareja, familiar y grupal) y enfoques psicoterapéuticos (psicodinámica, conductual-cognitiva, humanista, construccionista). Las sesiones fueron realizadas en su mayoría una vez por semana. Todas las terapias estuvieron a cargo de terapeutas con una vasta trayectoria profesional, quienes, al igual que los pacientes, dieron su consentimiento para participar en este estudio. Si bien los motivos de consulta fueron variados, las terapias coinciden en cuanto a que la problemática central de los consultantes se encuentra en el ámbito de las relaciones interpersonales.
En los 15 procesos terapéuticos se identificaron 331 episodios, de los cuales 215 corresponden a Episodios de Cambio y 116 a Episodios de Estancamiento. Estos episodios, insertos en 294 sesiones, constituyen el universo a partir del cual se seleccionó una muestra para ser sometida al posterior análisis a nivel de turno de palabra.

El muestreo de episodios consideró ambos tipos de episodio y se realizó de acuerdo al siguiente criterio metodológico: se seleccionaron tres Episodios de Cambio cuyos contenidos se ajustaran a cada uno de los tres niveles de la lista de Indicadores de Cambio Genéricos (Krause et al., 2007) y a cada una de las fases de la terapia (inicial, media y final). Por lo tanto, para cada una de las terapias analizadas fueron seleccionados: un Episodio de Cambio de la fase inicial de la terapia, que simultáneamente correspondiese al Nivel I en la secuencia de Indicadores de Cambio (Consolidación inicial de la estructura de la relación terapéutica), un Episodio de Cambio de la fase media de la terapia, que correspondiese al Nivel II en los Indicadores (Aumento de la permeabilidad hacia nuevos entendimientos) y un Episodio de Cambio de la fase final del proceso, que correspondiese al Nivel III de los Indicadores (Construcción y consolidación de nuevos entendimientos). En el caso de los Episodios de Estancamiento, también se seleccionaron tres, uno por cada fase de la terapia, incluyendo aquél que estuviera en la misma sesión o una sesión cercana al Episodio de Cambio previamente seleccionado. Hubo algunos casos en que no fue posible seleccionar episodios debido a que no se cumplía con el criterio metodológico establecido (por ejemplo, no

Tabla 1. Descripción de los niveles de análisis que conforman la muestra

\begin{tabular}{|c|c|c|c|c|c|c|c|c|c|}
\hline \multirow[b]{3}{*}{$\mathrm{N}^{\circ}$} & \multirow[b]{3}{*}{ Enfoque terapéutico } & \multirow[b]{3}{*}{ Pacientes } & \multirow[b]{3}{*}{ Sesiones } & \multicolumn{6}{|c|}{ Tipos de Episodio } \\
\hline & & & & \multicolumn{3}{|c|}{ Episodios de Cambio } & \multicolumn{3}{|c|}{ Episodios Estancamiento } \\
\hline & & & & Total & Seleccionados & $\begin{array}{c}\text { Turnos de } \\
\text { Palabra }\end{array}$ & Total & Seleccionados & $\begin{array}{c}\text { Turnos de } \\
\text { Palabra }\end{array}$ \\
\hline 1 & Psicodinámica & 1 & 23 & 10 & 3 & 198 & 15 & 3 & 82 \\
\hline 2 & Psicodinámica & 1 & 18 & 14 & 3 & 79 & 7 & 2 & 85 \\
\hline 3 & Construccionista & 3 & 20 & 12 & 2 & 192 & 14 & 3 & 137 \\
\hline 4 & Grupal & 6 & 18 & 9 & 2 & 121 & - & - & - \\
\hline 5 & Psicodinámica & 1 & 21 & 24 & 3 & 44 & 12 & 3 & 53 \\
\hline 6 & Construccionista & 3 & 10 & 12 & 2 & 41 & 5 & 2 & 106 \\
\hline 7 & Grupal & 18 & 19 & 19 & 2 & 160 & - & - & - \\
\hline 8 & Humanista & 2 & 18 & 20 & 2 & 67 & 12 & 3 & 117 \\
\hline 9 & Humanista & 2 & 19 & 19 & 2 & 35 & 14 & 3 & 96 \\
\hline 10 & Psicodinámica & 1 & 20 & 22 & 3 & 269 & 12 & 3 & 540 \\
\hline 11 & Gestalt & 1 & 61 & 5 & 1 & 77 & 2 & 2 & 212 \\
\hline 12 & Cognitivo-Conductual & 1 & 6 & 13 & 3 & 133 & 2 & 2 & 54 \\
\hline 13 & Cognitivo-Conductual & 1 & 6 & 11 & 1 & 16 & 8 & 3 & 141 \\
\hline 14 & Psicodinámica & 1 & 20 & 11 & 2 & 91 & 4 & 2 & 113 \\
\hline 15 & Psicodinámica & 1 & 15 & 14 & 2 & 50 & 9 & 3 & 71 \\
\hline
\end{tabular}


existía un episodio con Indicador de Cambio de Nivel III en la tercera fase de la terapia), así como tampoco se identificaron Episodios de Estancamiento en algunos procesos psicoterapéuticos (terapias grupales) (ver Tabla 1).

Siguiendo estos criterios, se seleccionaron 33 Episodios de Cambio y 34 Episodios de Estancamiento. Al interior de estos episodios se seleccionó la totalidad de los turnos de palabra de pacientes y terapeutas, obteniéndose 1573 turnos de palabra correspondientes a Episodios de Cambio y 1807 turnos de palabra correspondientes a Estancamiento.

\section{Procedimiento}

Selección y Delimitación de Episodios de Cambio y Estancamiento. Los procesos terapéuticos fueron registrados en audio y video y transcritos textualmente. Los Episodios de Cambio y de Estancamiento fueron determinados por al menos dos jueces independientes entrenados en la identificación y delimitación de los mismos. El Episodio de Cambio se identifica a partir de un momento de cambio (Krause et al., 2006; Krause et al., 2007), que marca su final. Este momento de cambio debe cumplir con los criterios de: correspondencia teórica (coincidencia con un indicador de cambio), novedad, actualidad y consistencia (Krause et al., 2007). Para determinar el inicio del momento de cambio, ya definida la temática del mismo según el indicador genérico de cambio, se rastrea la interacción terapéutica hacia atrás en el tiempo, a fin de marcar el inicio del trabajo en dicha temática específica. Ese punto se define como el comienzo del episodio de cambio.

Por su parte, el Episodio de Estancamiento coincide con períodos de tiempo, durante la sesión, en los cuales aparece una detención temporal del proceso de cambio del paciente, debido a una reedición de su problema, o a una reiteración de patrones de funcionamiento ligados a éste. Así, los Episodios de Estancamiento se caracterizan por la falta de una construcción de nuevos significados (Herrera et al., 2009). Para su selección, los Episodios de Estancamiento deben coincidir con los criterios de correspondencia teórica (temática de estancamiento), actualidad y congruencia no verbal y, además, cumplir con los siguientes criterios metodológicos: tener una duración mínima de tres minutos y estar a una distancia de por lo menos 10 minutos de algún Episodio de Cambio.

Análisis de las Acciones Comunicacionales. Se utilizó el Sistema de Codificación de la Actividad Terapéutica (SCAT-1.0, Valdés et al., 2010), previamente descrito, para codificar las verbalizaciones de pacientes y terapeutas, presentes en cada turno de palabra de los Episodios de Cambio y los Episodios de Estancamiento. Como ya se señalara, este sistema permite distinguir entre: a) Formas Básicas, referidas a la estructura formal de la verbalización; b) Intenciones Comunicacionales, que se refieren al propósito comunicacional de la verbalización; c) Técnicas, que se refieren a los recursos metodológicos utilizados para apoyar el propósito comunicacional; d) Ámbito, que indica si el objeto del trabajo terapéutico es principalmente cognitivo, afectivo o comportamental; y e) Referencia, que indica quién es el protagonista del trabajo terapéutico en la verbalización del hablante (ver Figura 1).

El SCAT-1.0 fue aplicado a los turnos de palabra del discurso de paciente y terapeuta -en los episodios seleccionados y transcritos- por duplas de codificadores, en dos etapas consecutivas. En la primera, cada investigador codificó en forma individual los episodios y, en la segunda etapa, las parejas de investigadores conciliaron sus codificaciones individuales de manera de obtener una codificación final plenamente acordada. Fueron estas codificaciones conciliadas las que se sometieron al posterior análisis cuantitativo.

\section{Análisis de Datos}

El análisis de los datos se realizó a través de un modelo de regresión logística con la intención de determinar si había diferencias en la probabilidad de presentación de las diferentes Acciones Comunicacionales según el tipo de episodio y el emisor del discurso, una vez controladas las variables tipo de terapia y fase de ésta en que se presentan los episodios.

El modelo de regresión logística consideró los predictores: Tipo de episodio, diferenciando entre los Episodios de Cambio y Estancamiento (esta última es la categoría de referencia, con valor "0" asignado); Emisor del discurso, distinguiendo si las verbalizaciones son emitidas por el paciente o terapeuta (categoría referencia); Tipo de terapia, cuyas categorías son: psicodinámica (categoría de referencia), humanista, cognitivo-conductual, gestáltica y otras, (que incluye terapias grupal y construccionista); Fase de la terapia, diferenciándose tres fases de similar longitud, denominadas: inicial (categoría de referencia), media y final, con base en el número total de sesiones de cada terapia; y la interacción entre Tipo de emisor y Tipo de episodio.

La ecuación de regresión es la siguiente:

$$
\operatorname{Logit}(y)=\beta_{\text {Tipo Terapia }}+\beta_{\text {Fase Terapia }}+\beta_{\text {Emisor }}+\beta_{\text {Tipo Episodio }}+\left[\beta_{\text {Emisor" tipo Episodio }}\right]
$$

Para llevar a cabo estos análisis se dicotomizó cada una de las dimensiones del SCAT-1.0. Por ejemplo, al considerar la Forma Básica Negar se codificó "1" cuando se presentaba y "0" cuando se presentaban las otras Formas Básicas disponibles.

En cada uno de los modelos de regresión estimados, se modeló el componente interacción entre el tipo de episodio y el emisor del discurso, controlando las covariables. En los casos en que dicha interacción fue significativa, sus resultados son reportados a través de la estimación de Odds Ratio (OR) y graficadas para su mejor comprensión ${ }^{3}$.

\footnotetext{
3 Los gráficos son sólo referenciales, ya que no consideran las variables control.
} 
La dimensión Técnicas del SCAT-1.0 fue analizada comparando los porcentajes de aparición, entre los Episodios de Cambio vs. Estancamiento, a través de chi-cuadrado, dada la baja frecuencia de algunas de sus categorías.

\section{Resultados}

\section{Formas Básicas}

Como se puede apreciar en la Tabla II, la Forma Básica más frecuente en ambos tipos de episodio fue Aseverar, seguida por Asentir en los Episodios de Cambio, y luego por Preguntar.

El modelo de regresión logística indica que la interacción entre Tipo de episodio* Emisor, $\chi 2(1)=55,29, p \leq$ .001 , predice en forma significativa la probabilidad de aparición de la Forma Básica Asentir. Así, en los Episodios de Estancamiento el terapeuta asiente en forma más frecuente que el paciente $(\mathrm{OR}=.73 ; 95 \% \mathrm{IC}=.58-.90)$, a diferencia de los Episodios de Cambio, donde es el paciente el que
Asiente con mayor frecuencia que el terapeuta $(\mathrm{OR}=2.50$; $95 \%$ IC $=1.96-3.20$; ver Figura 2).

Similares resultados se presentan al considerar el uso de la Forma Básica Aseverar. El modelo de regresión logística indica que la interacción entre Tipo de Episodio* Emisor es significativa, $\chi 2(1)=16.25, p \leq .001$, indicando que en los Episodios de Estancamiento el paciente Asevera más frecuentemente que el terapeuta $(\mathrm{OR}=2.25 ; 95 \% \mathrm{IC}=$ 1.87 - 2.73), a diferencia de los Episodios de Cambio, en los que la diferencia no es $\tan$ marcada $(\mathrm{OR}=1.3 ; 95 \% \mathrm{IC}=$ 1.06 - 1.60) (ver Figura 3).

Al considerar el uso de la Forma Básica Negar, los resultados indican que la interacción entre Tipo de Episodio* Emisor es significativa, $\chi 2(1)=35.33, p \leq .001$, indicando que en los Episodios de Estancamiento el paciente Niega en forma más frecuente que el terapeuta $(\mathrm{OR}=4.2 ; 95 \%$ $\mathrm{IC}=2.47$ - 7.07), patrón que se invierte en el Episodio de Cambio $(\mathrm{OR}=.63 ; 95 \% \mathrm{IC}=.42-.94)$, siendo el terapeuta

Tabla 2. Frecuencias y porcentajes de uso de Formas Básicas según el tipo de episodio y el tipo de emisor

\begin{tabular}{lcccc}
\hline & \multicolumn{2}{c}{ Episodios de Cambio } & \multicolumn{2}{c}{ Episodios de Estancamiento } \\
\multicolumn{1}{c}{ Formas Básicas } & Pacientes & Terapeutas & Pacientes & Terapeutas \\
\hline Asentir & $32.42(235)$ & $16.10(127)$ & $22.25(196)$ & $28.27(244)$ \\
Aseverar & $57.87(423)$ & $51.33(405)$ & $63.56(560)$ & $43.68(377)$ \\
Negar & $5.61(41)$ & $8.62(68)$ & $8.17(72)$ & $2.09(18)$ \\
Preguntar & $3.69(27)$ & $20.41(161)$ & $5.56(49)$ & $24.22(209)$ \\
Dirigir & $0.41(3)$ & $3.55(28)$ & $0.45(4)$ & $1.74(15)$ \\
Total $(f)$ & 731 & 789 & 863 & 881 \\
\hline
\end{tabular}

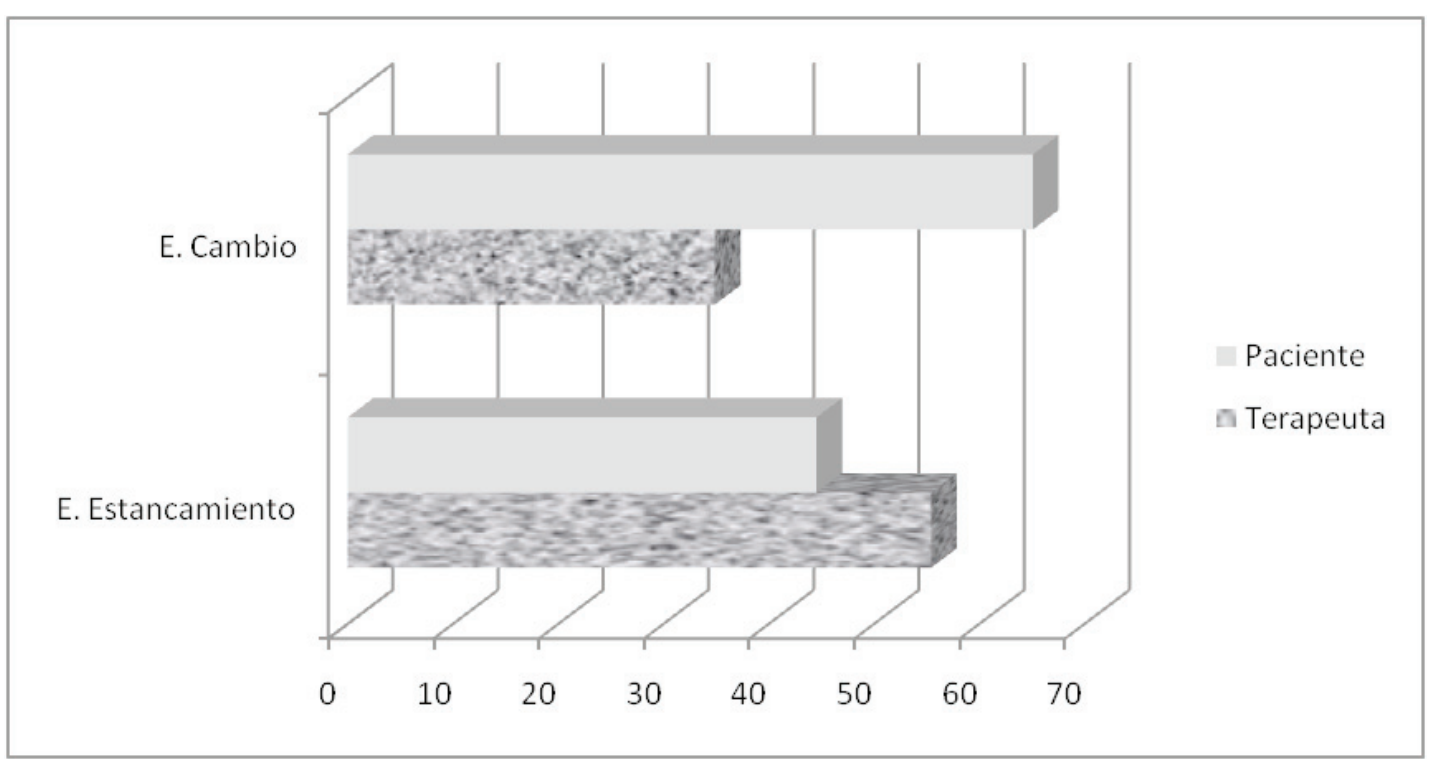

Figura 2. Uso de la Forma Básica Asentir en Episodios de Cambio y Estancamiento, según el rol (Pacientes y Terapeutas). 


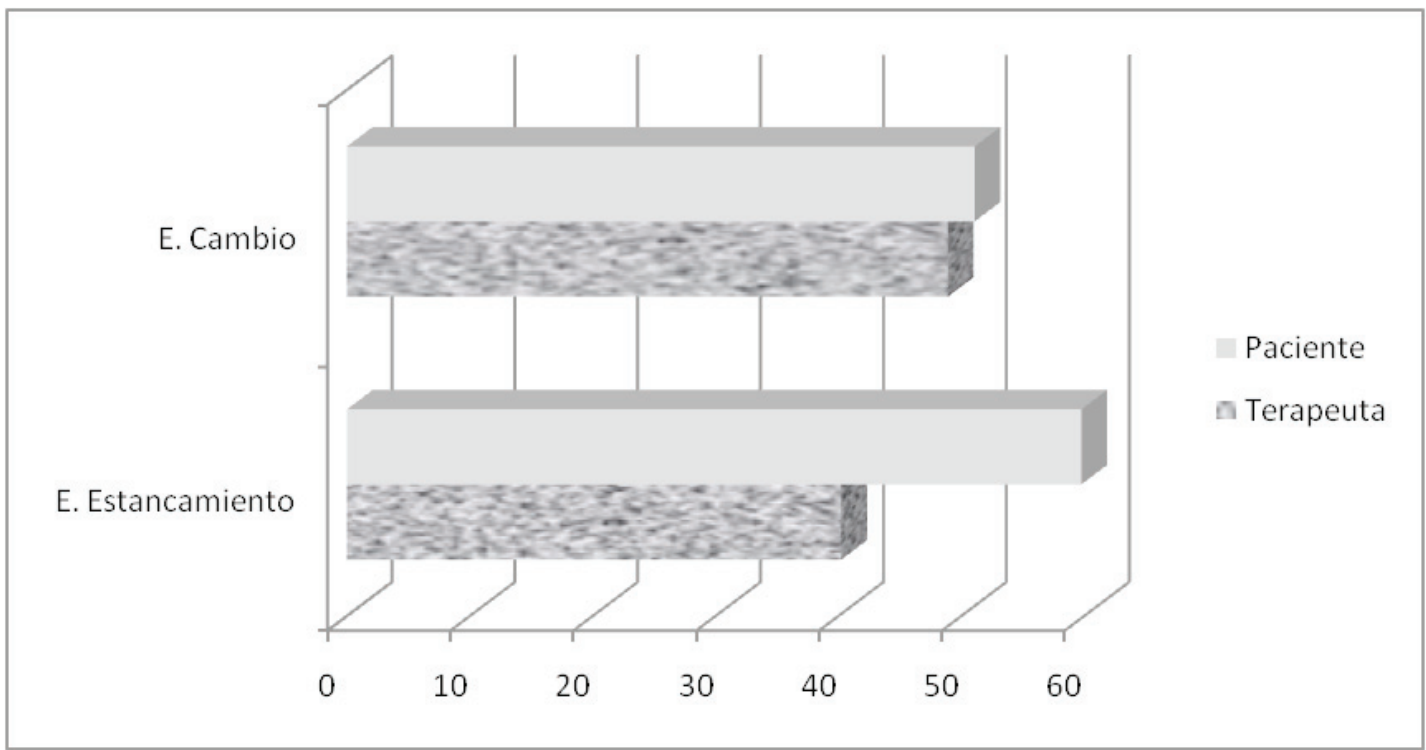

Figura 3. Uso de la Forma Básica Aseverar en Episodios de Cambio y Estancamiento, según el rol (Pacientes y Terapeutas).

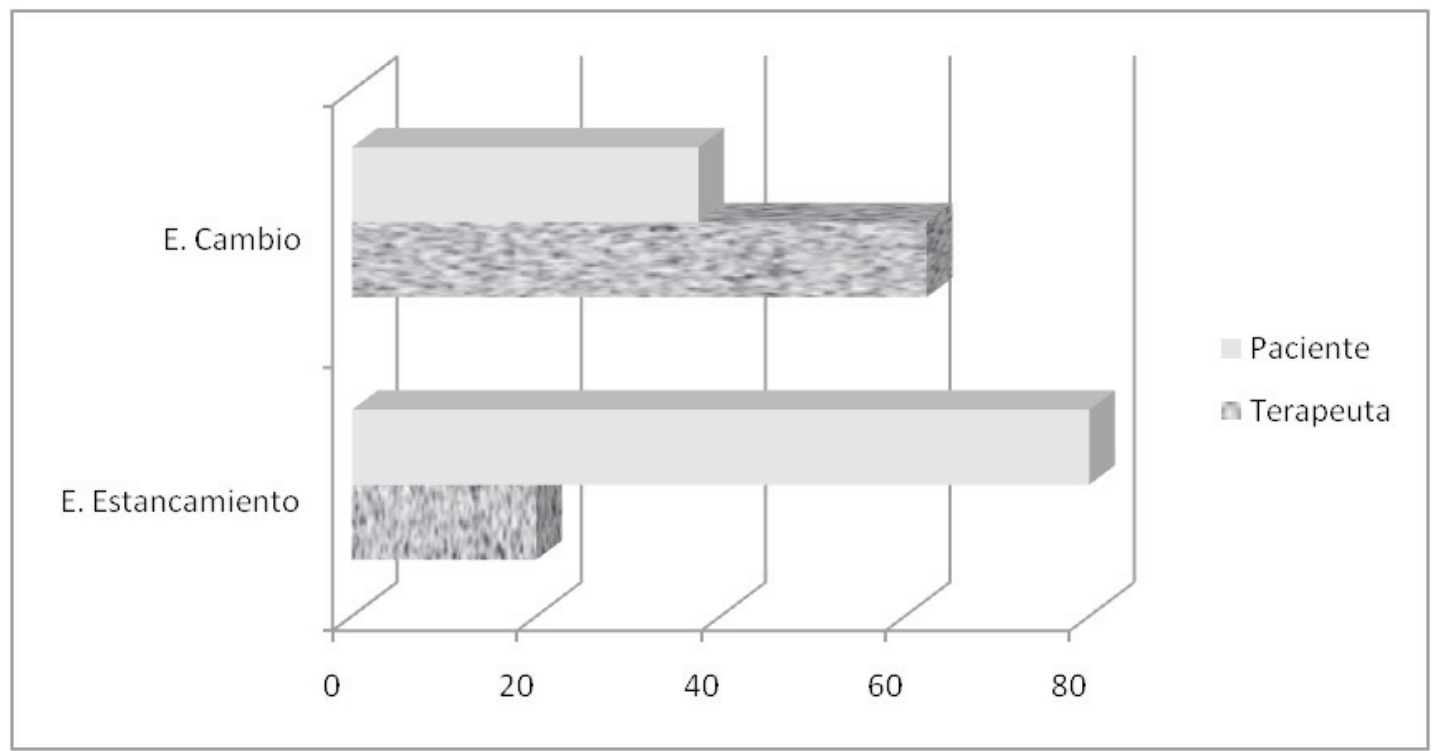

Figura 4. Uso de la Forma Básica Negar en Episodios de Cambio y Estancamiento, según el rol (Pacientes y Terapeutas).

el que Niega en forma más frecuente que el paciente (Ver Figura 4).

Finalmente, respecto al uso de la Forma Básica Preguntar, los resultados indican que el Tipo de episodio $(p \leq .001)$ y el Emisor $(p \leq .001)$, permiten predecir su probabilidad de uso. Así, es más probable preguntar en los Episodios de Estancamiento (comparado con los de cambio, $\mathrm{OR}=.65 ; 95 \% \mathrm{IC}=.52-.81)$, y por parte de los terapeutas (que por parte de los pacientes, $\mathrm{OR}=.16 ; 95 \%$ $\mathrm{IC}=.12-.21)$, sin presentarse interacción significativa entre estos componentes $(p>.05)$.

\section{Intenciones Comunicacionales}

A continuación se presentan los resultados relativos al uso de las diferentes Intenciones Comunicacionales, por parte de pacientes y terapeutas, en Episodios de Cambio y de Estancamiento. Como se aprecia en la Tabla III, la de mayor frecuencia es la Intención Comunicacional Explorar, en ambos hablantes y ambos Tipos de episodio, con un predominio en los Episodios de Estancamiento. Los predominios de las Intenciones Comunicacionales Sintonizar y Resignificar dependen del Tipo de emisor y del Tipo de episodio. 
Tabla 3. Frecuencia de uso de las Intenciones Comunicacionales según tipo de Episodio y tipo de Emisor.

\begin{tabular}{ccccc}
\hline & \multicolumn{2}{c}{ Episodios de Cambio } & \multicolumn{2}{c}{ Episodios de Estancamiento } \\
$\begin{array}{c}\text { Intenciones } \\
\text { Comunicacionales }\end{array}$ & Pacientes & Terapeutas & Pacientes & Terapeutas \\
\hline Explorar & $53.56(241)$ & $30.62(188)$ & $74.18(454)$ & $39.54(225)$ \\
Sintonizar & $16.00(72)$ & $29.64(182)$ & $22.55(138)$ & $32.51(185)$ \\
Resignificar & $30.44(137)$ & $39.74(244)$ & $3.27(20)$ & $27.94(159)$ \\
Total $(f)$ & 450 & 614 & 612 & 569 \\
\hline
\end{tabular}

Tabla 4. Frecuencia de uso de las Técnicas Terapéuticas según el tipo de episodio y el tipo de emisor

\begin{tabular}{|c|c|c|c|c|}
\hline \multirow[b]{2}{*}{ Técnicas } & \multicolumn{2}{|c|}{ Episodios de Cambio } & \multicolumn{2}{|c|}{ Episodios de Estancamiento } \\
\hline & Pacientes & Terapeutas & Pacientes & Terapeutas \\
\hline Argumentación & $79.61(121)$ & $12.90(28)$ & 79.18 (194) & $17.21(37)$ \\
\hline Auto-revelación ${ }^{a}$ & $1.97(3)$ & $9.22(20)$ & $1.22(3)$ & $1.40(3)$ \\
\hline Confrontación & $0.00(0)$ & $10.60(23)$ & $3.67(9)$ & $23.72(51)$ \\
\hline Consejo $^{a}$ & $4.61(7)$ & $11.98(26)$ & $0.00(0)$ & $3.26(7)$ \\
\hline Imaginería $^{\mathrm{a}}$ & $0.00(0)$ & $3.23(7)$ & $0.00(0)$ & $0.00(0)$ \\
\hline Información ${ }^{a}$ & $0.00(0)$ & $5.07(11)$ & $0.00(0)$ & $3.26(7)$ \\
\hline Interpretación & $2.63(4)$ & $17.97(39)$ & $0.41(1)$ & $26.05(56)$ \\
\hline Narración ${ }^{a}$ & $1.97(3)$ & $0.00(0)$ & $11.84(29)$ & $0.47(1)$ \\
\hline Nominación $^{\mathrm{a}}$ & $6.58(10)$ & $2.30(5)$ & $3.67(9)$ & $6.51(14)$ \\
\hline Paradoja $^{\mathrm{a}}$ & $0.00(0)$ & $1.38(3)$ & $0.00(0)$ & $1.86(4)$ \\
\hline Reflejo & $0.00(0)$ & $15.21(33)$ & $0.00(0)$ & $11.16(24)$ \\
\hline Refuerzo $^{\text {a }}$ & $1.97(3)$ & $5.53(12)$ & $0.00(0)$ & $0.93(2)$ \\
\hline Resumen $^{\text {a }}$ & $0.66(1)$ & $3.69(8)$ & $0.00(0)$ & $2.33(5)$ \\
\hline Role Playing ${ }^{a}$ & $0.00(0)$ & $0.92(2)$ & $0.00(0)$ & $1.86(4)$ \\
\hline Total $(f)$ & 152 & 215 & 245 & 217 \\
\hline
\end{tabular}

Nota. a Técnicas agrupadas bajo categoría "Otros” en el análisis chi-cuadrado.

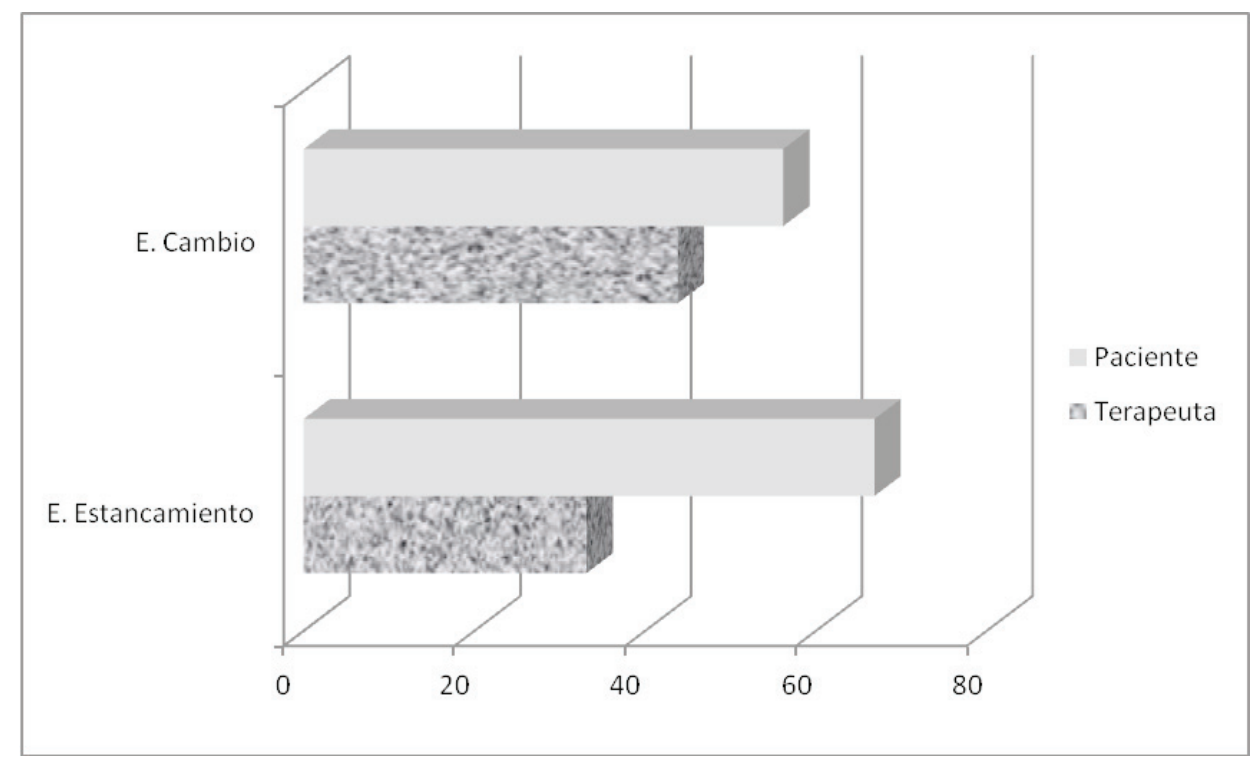

Figura 5. Uso de la Intención Comunicacional Explorar en Episodios de Cambio y Estancamiento, según el rol (Pacientes y Terapeutas). 


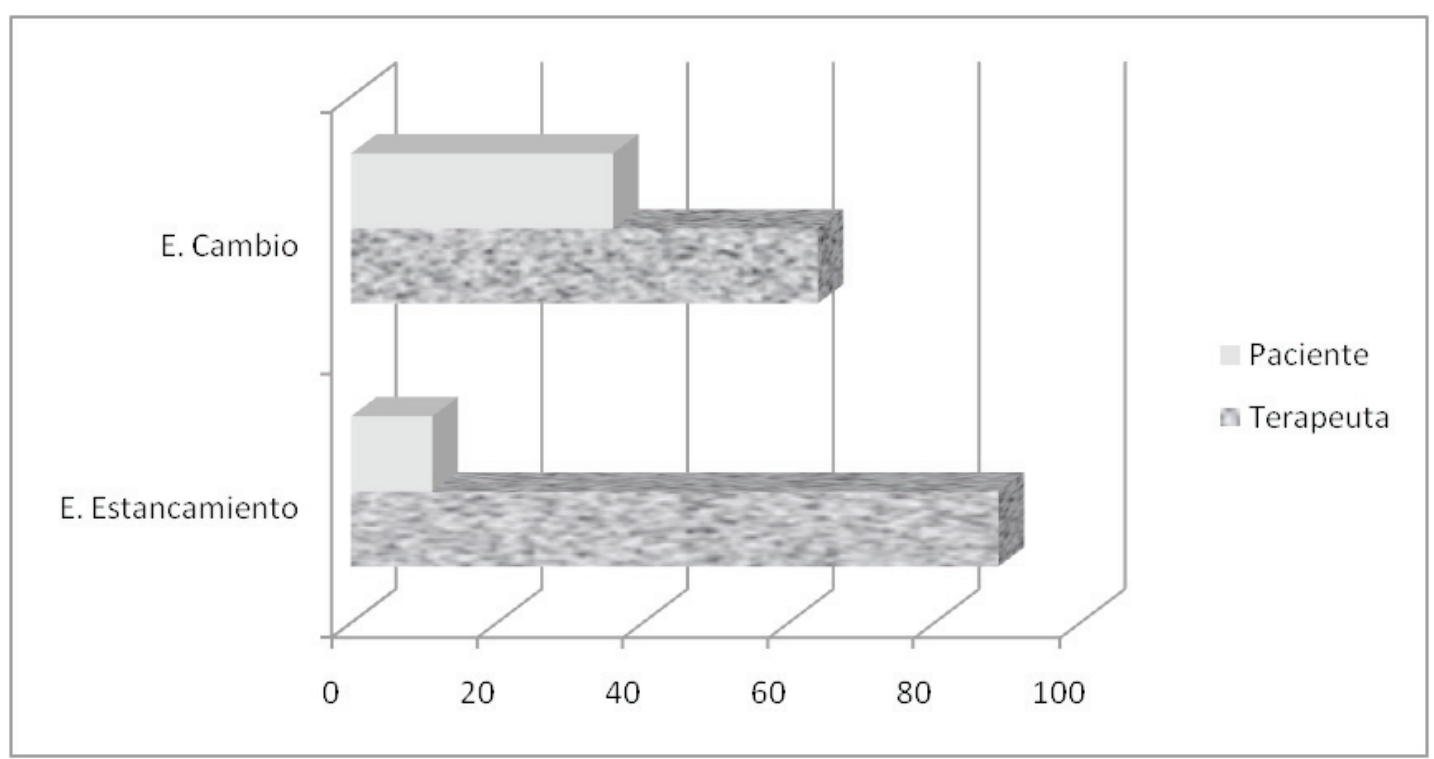

Figura 6. Uso de la Intención Comunicacional Resignificar en Episodios de Cambio y Estancamiento, según el rol (Pacientes y Terapeutas).

Tabla 5. Frecuencias relativas al Ámbito según el tipo de episodio y el emisor

\begin{tabular}{lcccc}
\hline & \multicolumn{2}{c}{ Episodios de Cambio } & \multicolumn{2}{c}{ Episodios de Estancamiento } \\
\multicolumn{1}{c}{ Ámbito } & Pacientes & Terapeutas & Pacientes & Terapeutas \\
\hline Acciones & $24.82(101)$ & $20.63(111)$ & $27.12(166)$ & $18.10(103)$ \\
Ideas & $56.51(230)$ & $65.61(353)$ & $54.41(333)$ & $60.98(347)$ \\
Afectos & $18.67(76)$ & $13.75(74)$ & $18.46(113)$ & $20.91(119)$ \\
Total $(f)$ & 407 & 538 & 612 & 569 \\
\hline
\end{tabular}

El modelo de regresión confirma que el Tipo de Episodio $(p<.001)$ y el Emisor $(p<.001)$ predicen en forma significativa el uso de la Intención Comunicacional Explorar, luego de controlar el Tipo de terapia $(p=.12)$ y las Fases de ésta $(p<.001)$. Adicionalmente, la interacción entre Tipo de Episodio*Emisor es significativa, $\left(\chi^{2}(1)=11.235, p \leq\right.$ $.001)$, indicando que en los Episodios de Estancamiento el paciente Explora más que el terapeuta $(\mathrm{OR}=4.39 ; 95 \%$ $\mathrm{IC}=3.43$ - 5.62), y que en el Episodio de Cambio esta diferencia es menos marcada $(\mathrm{OR}=2.61 ; 95 \% \mathrm{IC}=2.3-$ 3.36; ver Figura 5).

Respecto de la Intención Comunicacional Resignificar, el modelo de regresión logística indica que el Tipo de episodio $(p \leq .001)$ y el Emisor $(p \leq .001)$ predicen la probabilidad de utilizar esta Intención Comunicacional, luego de controlar el Tipo de terapia $(p=.022)$ y las Fases de ésta $(p=.11)$. Asimismo, la interacción entre Tipo de Episodio*Emisor fue significativa, $\left(\chi^{2}(1)=63.75, p \leq\right.$ $.001)$, indicando que en los Episodios de Estancamiento el terapeuta Resignifica más frecuentemente que el paciente $(\mathrm{OR}=.09 ; 95 \% \mathrm{IC}=.05-.14)$, mientras que en el Episodio de Cambio, dicha diferencia no es tan marcada $(\mathrm{OR}=.66$; $95 \% \mathrm{IC}=.51-.86)($ Figura 6$)$.
Finalmente, al considerar la Intención Comunicacional Sintonizar, el modelo de regresión logística indica que el Tipo de episodio $(p \leq .001)$ y el Emisor $(p \leq .001)$ predicen su probabilidad de aparición (luego de controlar las variables). Es más probable Sintonizar en los Episodios de Estancamiento (que en los de cambio; OR=.70; 95\% $\mathrm{IC}=.58-.86) \mathrm{y}$, considerando ambos Tipos de episodio, los terapeutas tienen mayor probabilidad de Sintonizar que los pacientes $(\mathrm{OR}=.55 ; 95 \% \mathrm{IC}=.45-.67)$. Este patrón es constante, ya que no se presenta interacción significativa entre estos componentes $(p>.05)$.

\section{Técnicas Comunicacionales}

Los resultados muestran que las Técnicas de mayor frecuencia de aparición son Argumentación, Confrontación, Interpretación y Reflejo, presentándose diferencias estadísticamente significativas en el uso de ellas, de acuerdo al Tipo de episodio $\left(\chi^{2}(4, N=432)=10.93, p \leq .03\right)$. La Tabla IV presenta el uso de las diferentes Técnicas por parte del terapeuta en los Episodios de Cambio y Estancamiento. Al comparar las frecuencias de las Técnicas (agrupadas en Argumentación, Confrontación, Interpretación, Reflejo y Otros, dada la baja frecuencia de algunas de ellas) entre ambos tipos de episodio, 


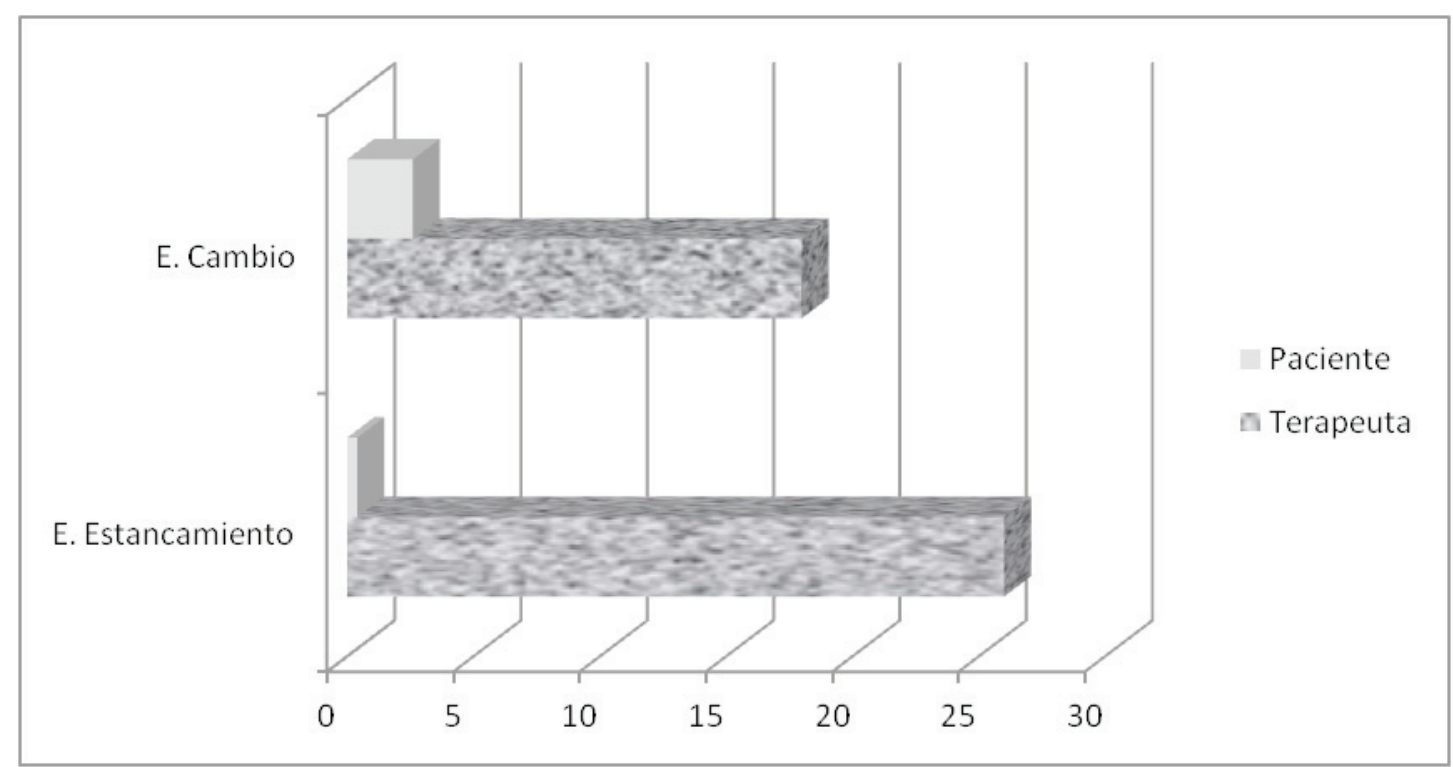

Figura 7. Uso de la Técnica Interpretación en Episodios de Cambio y Estancamiento, según el rol (Pacientes y Terapeutas).

Tabla 6. Frecuencias relativas a la Referencia según el tipo de episodio y el emisor

\begin{tabular}{lcccc}
\hline \multicolumn{1}{c}{ Referencia } & \multicolumn{2}{c}{ Episodios de Cambio } & \multicolumn{2}{c}{ Episodios de Estancamiento } \\
& Pacientes & Terapeutas & Pacientes & Terapeutas \\
\hline Sí mismo & $57.99(236)$ & $7.62(41)$ & $49.51(303)$ & $5.27(30)$ \\
El otro presente & $5.65(23)$ & $53.16(286)$ & $5.39(33)$ & $52.20(297)$ \\
Terceros (no presente) & $5.41(22)$ & $4.46(24)$ & $14.71(90)$ & $8.96(51)$ \\
Relación terapéutica & $5.41(22)$ & $10.22(55)$ & $8.99(55)$ & $11.60(66)$ \\
Relación con un tercero & $12.78(52)$ & $7.06(38)$ & $12.75(78)$ & $7.03(40)$ \\
Neutro & $12.78(52)$ & $17.47(94)$ & $8.66(53)$ & $14.94(85)$ \\
Total (f) & 407 & 538 & 612 & 569 \\
\hline
\end{tabular}

Tabla 7. Resumen de diferencias entre Episodios de Cambio y Estancamiento

\begin{tabular}{|c|c|c|}
\hline & Episodios de Cambio & Episodios de Estancamiento \\
\hline $\begin{array}{l}\text { ¿Qué están haciendo en } \\
\text { términos de forma verbal? }\end{array}$ & $\begin{array}{l}\text { El terapeuta tiene un discurso más propositivo (ase- } \\
\text { vera y niega más; asiente menos); el paciente tiene un } \\
\text { lenguaje más receptivo, asintiendo más. }\end{array}$ & $\begin{array}{l}\text { El paciente asevera y niega más, y asiente menos. } \\
\text { Paciente con discurso más cerrado, poco receptivo. } \\
\text { El terapeuta asiente más, y niega y asevera menos. }\end{array}$ \\
\hline $\begin{array}{l}\text { ¿Con qué intención } \\
\text { comunicacional? }\end{array}$ & Terapeuta y paciente buscan nuevos significados. & $\begin{array}{l}\text { El terapeuta intenta buscar nuevos significados, pero } \\
\text { el paciente mantiene sus narrativas sin modificar. } \\
\text { Hay mayor diferencia entre lo que ambos buscan. }\end{array}$ \\
\hline $\begin{array}{l}\text { ¿Con qué técnicas } \\
\text { comunicacionales? }\end{array}$ & $\begin{array}{l}\text { Los terapeutas usan más variedad de técnicas que } \\
\text { los pacientes, los que utilizan principalmente la } \\
\text { Argumentación. }\end{array}$ & $\begin{array}{l}\text { Los terapeutas usan más la confrontación y la inter- } \\
\text { pretación que en los Episodios de Cambio. } \\
\text { Los pacientes utilizan principalmente la } \\
\text { Argumentación. }\end{array}$ \\
\hline ¿De qué ámbito se habla? & $\begin{array}{l}\text { Predominan las ideas, pero el paciente se enfoca más } \\
\text { en los afectos que el terapeuta. }\end{array}$ & $\begin{array}{l}\text { Predominan las ideas. Ambos no se distinguen en } \\
\text { cuanto al foco en los afectos. }\end{array}$ \\
\hline $\begin{array}{l}\text { ¿Acerca de quién están } \\
\text { hablando? }\end{array}$ & Ambos hablan predominantemente del paciente. & $\begin{array}{l}\text { Se habla más acerca de terceras personas y de } \\
\text { la relación terapéutica (que en los Episodios de } \\
\text { Cambio). }\end{array}$ \\
\hline
\end{tabular}




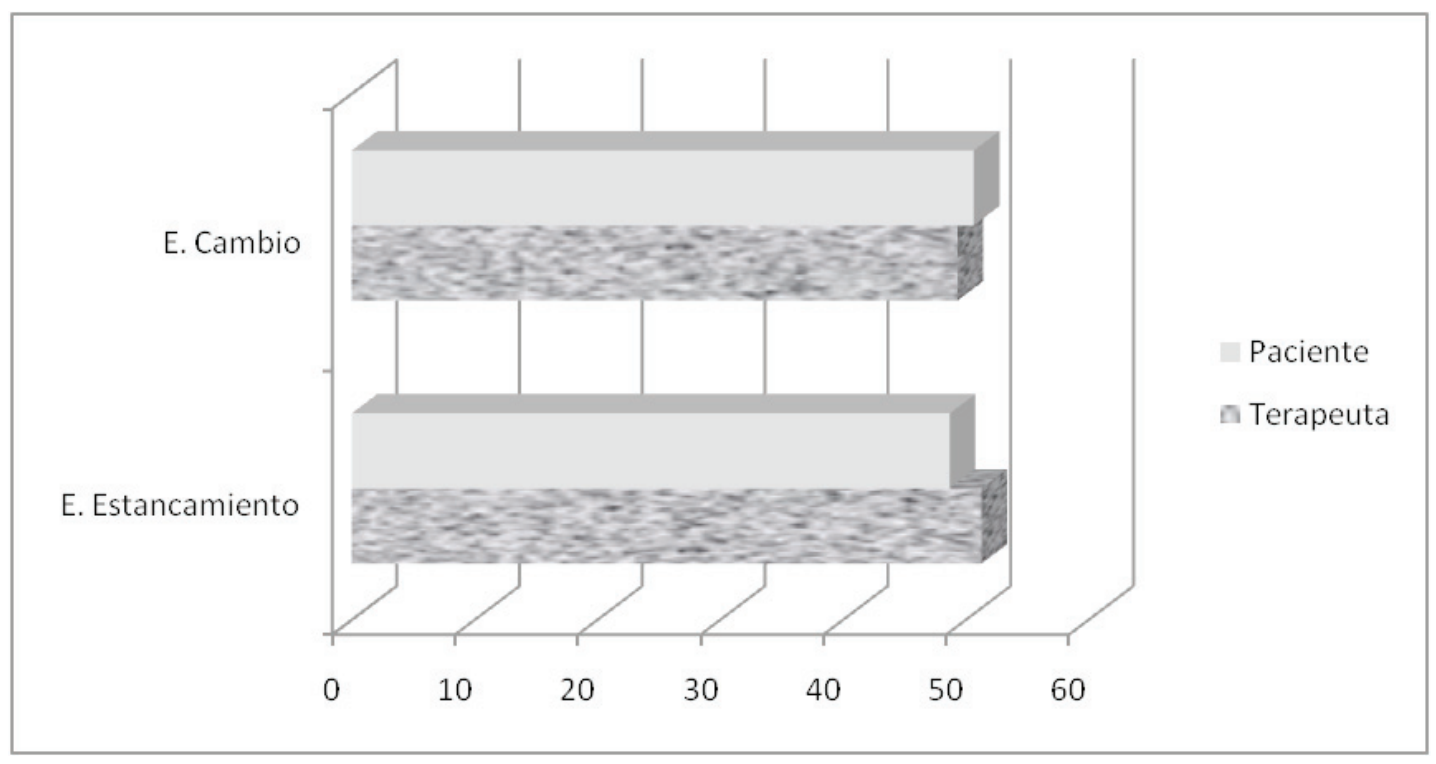

Figura 8. Uso del Ámbito Afectos en Episodios de Cambio y Estancamiento, según el rol (Pacientes y Terapeutas).

los resultados indican que se presentan diferencias estadísticamente significativas $\left(\chi^{2}(4, \mathrm{~N}=432)=10.93, p \leq .03\right)$. En los Episodios de Estancamiento es más frecuente el uso de la Confrontación $(p \leq .001)$ y la Interpretación $(\mathrm{p} \leq .05)$, en contraste con los Episodios de Cambio, donde es más frecuente el uso de las otras técnicas terapéuticas $(p \leq .001)$ como, por ejemplo, la Autorrevelación o el Consejo.

El análisis de regresión se desarrolló con las tres Técnicas más frecuentes: Argumentación, Confrontación e Interpretación. El modelo de regresión indica que sólo el Emisor $(p<.001)$ predice en forma significativa el uso de la Argumentación en la interacción verbal terapéutica, luego de controlar el Tipo de terapia $(p=.03)$ y las Fases de ésta $(p=.69)$. No hay diferencias en su aparición según el Tipo de Episodio $(p=.37)$. Independientemente del tipo de episodio es más probable que el paciente Argumente más que el terapeuta $(\mathrm{OR}=22.04 ; 95 \% \mathrm{IC}=15.37-32.64)$.

En cuanto a la Técnica Confrontación, tanto el Tipo de Episodio $(p<.001)$ como el Emisor $(p<.001)$ dan cuenta del uso de ella, luego de controlar por las variables Tipo de terapia $(p=.11)$ y Fases $(p=.72)$. En este caso es más probable que la Confrontación sea más usada por el terapeuta $(\mathrm{OR}=.09 ; 95 \% \mathrm{IC}=.05-.19)$. Respecto de los Tipos de episodio, es más probable que ambos actores utilicen la Confrontación en los Episodios de Estancamiento ( $\mathrm{OR}=.29$; $95 \% \mathrm{IC}=.16-.51)$ que en los Episodios de Cambio. Estos patrones son constantes, ya que no se presentan interacciones significativas entre Emisor y el Tipo de Terapia $(p>.05)$.

Finalmente, respecto del uso de la Interpretación, los resultados indican que sólo el Emisor $(p<.001)$ predice su uso (no así el Tipo de Episodio, $p=.16$ ), luego de controlar el Tipo de terapia $(p<.001)$ y las Fases de ésta $(p=.91)$. Sin embargo, la interacción entre Tipo de Episodio*Emisor es significativa, $\left(\chi^{2}(1)=5.902, p \leq .02\right)$, indicando que en los Episodios de Estancamiento el terapeuta Interpreta más que el paciente $(\mathrm{OR}=.01 ; 95 \% \mathrm{IC}=.01-.09)$, y que en el Episodio de Cambio esta diferencia es menos marcada $(\mathrm{OR}=.12 ; 95 \% \mathrm{IC}=.04-.35)($ ver Figura 7$)$.

\section{Ámbito}

Como se señaló anteriormente, la categoría Ámbito dentro del SCAT-1.0 corresponde a una de las dos dimensiones de Contenido de la Acciones Comunicacionales. Específicamente, permite clasificar el Contenido sobre el cual se está focalizando el trabajo terapéutico, distinguiendo si el objeto del trabajo terapéutico se sitúa principalmente en el plano comportamental (ámbito de las acciones), $\operatorname{cog}$ nitivo (ámbito Ideas), o emocional (ámbito de los afectos).

En términos generales, e independientemente del Tipo de episodio, el discurso terapéutico se centra más en el Ámbito de las Ideas, luego en las Acciones y, finalmente, en las Emociones, tanto en los Episodios de Cambio como de Estancamiento (ver Tabla V).

Los resultados de la regresión logística indican que el Tipo de episodio no predice en forma significativa el Ámbito de las Ideas $(p=.09)$, al controlar el Tipo de terapia ( $p \leq$ $.001)$ y las Fases de ésta $(p=.43)$, en tanto el Emisor del discurso sí es un predictor significativo $(p \leq .001)$. Así, la probabilidad de referirse al Ámbito de las Ideas es similar en los Episodios de Cambio y Estancamiento (OR=1.17; 95\% $\mathrm{IC}=.98-1.40$ ), pero es más probable que los terapeutas se focalicen en las Ideas (comparado con los pacientes, $(\mathrm{OR}=$ $.72 ; 95 \% \mathrm{IC}=.61-.87$ ). Algo similar ocurre en el Ámbito de las Acciones, donde el Tipo de episodio no predice su ocurrencia $(p=.86)$, pero el Emisor del discurso sí lo hace $(p \leq .001)$, siendo probable que los pacientes se focalicen 
en las Acciones más que los terapeutas ( $\mathrm{OR}=1.55 ; 95 \%$ $\mathrm{IC}=1.26-1.91)$. Estos patrones son constantes, ya que no se presentan interacciones $(p>.05)$.

Un patrón diferente se presenta en relación con el Ámbito de las Emociones. Los resultados indican que si bien el Tipo de episodio $(p=.05)$ y el Emisor $(p=.97)$ no son predictores significativos, al controlar las Fases de la terapia $(p \leq .01)$ y su Tipo $(p<.001)$, la interacción entre ellos sí lo es (Tipo de Episodio* Emisor, $\chi 2(1)=5.98, p \leq .05$ ). Se observa, entonces, que en los Episodios de Estancamiento, terapeutas y pacientes se focalizan con similar frecuencia en las Emociones $(\mathrm{OR}=.86 ; 95 \% \mathrm{IC}=.64-.14)$, en contraste con los Episodios de Cambio, donde los pacientes se focalizan más frecuentemente en las Emociones que los terapeutas $(\mathrm{OR}=1.44 ; 95 \% \mathrm{IC}=1.02-2.04)$ (ver Figura 8$)$.

\section{Referencia}

La categoría Referencia indica acerca de quién se está hablando. Tal y como se aprecia en la Tabla VI, las Referencias más frecuentes son a sí mismo y al otro presente, apreciándose diferencias según actor y Tipo de episodio.

Los análisis de regresión logística indican que, al controlar las variables Tipo de terapia y Fase de la terapia, en los Episodios de Estancamiento los Emisores se Refieren más a un tercero $(p \leq .001 ; \mathrm{OR}=.40 ; 95 \% \mathrm{IC}=.28-.58)$ y a la relación terapéutica $(p \leq .01 ; \mathrm{OR}=.63 ; 95 \% \mathrm{IC}=.46-$ .87), a diferencia de los Episodios de Cambio, en los cuales es más probable que se hable de sí mismo ( $p \leq .001 ; \mathrm{OR}=$ $1.50 ; 95 \% \mathrm{IC}=1.19-1.90)$, y se utilice una Referencia de carácter neutral $(p \leq .001 ; \mathrm{OR}=1.61 ; 95 \% \mathrm{IC}=1.22-2.13)$.

Adicionalmente (también controlando las variables Tipo de terapia y Fase de la terapia), se presentan diferencias en la probabilidad de uso de estas Referencias según el Emisor de las verbalizaciones. Así, los pacientes tienen mayor probabilidad de Referirse a sí mismos ( $p \leq .001$; $\mathrm{OR}=17.99 ; 95 \% \mathrm{IC}=13.63-23.77)$, a un tercero $(p \leq$ $.01 ; \mathrm{OR}=1.61 ; 95 \% \mathrm{IC}=1.17-2.28)$, a la relación con un tercero $(p \leq .001 ; \mathrm{OR}=1.92 ; 95 \% \mathrm{IC}=1.42-2.59)$, $\mathrm{y}$ utilizar las Referencias neutrales ( $p \leq .01 ; \mathrm{OR}=1.47 ; 95 \%$ $\mathrm{IC}=1.11-1.94)$, en cambio los terapeutas tienen mayor probabilidad de Referirse al otro presente ( $p \leq .001$; OR $=.05 ; 95 \% \mathrm{IC}=.04-.07)$ y a la relación terapéutica $(p \leq$ $.001 ; \mathrm{OR}=.59 ; 95 \% \mathrm{IC}=.43-.81)$. Finalmente, ninguna de las interacciones modeladas entre Tipo de Episodio* Emisor fue estadísticamente significativa $(p>.05)$.

\section{Discusión}

\section{Sintesis de los resultados}

La pregunta central de este estudio fue: ¿Cuáles son las diferencias entre Episodios de Cambio y Estancamiento en cuanto al lenguaje utilizado por ambos actores durante el diálogo terapéutico? Los resultados presentados muestran que aparecen diferencias entre ambos tipos de episodio en cada una de las dimensiones evaluadas por el SCAT-1.0, vale decir, en el uso de Formas Básicas, en las Intenciones Comunicacionales presentes, en las Técnicas comunicacionales utilizadas, en el Dominio al que se refieren las verbalizaciones -las ideas, los afectos, o las acciones- y en cuanto a quien es el protagonista de la verbalización.

En lo que se refiere al uso de Formas Básicas, comparando al terapeuta con el consultante y los tipos de episodios, los resultados muestran que el terapeuta Asiente más en los Episodios de Estancamiento y el consultante lo hace más en los Episodios de Cambio. En cuanto al uso de Aseveraciones, siendo en general los pacientes los que aseveran más frecuentemente que los terapeutas, esta diferencia es más marcada en los Episodios de Estancamiento que en los de cambio, vale decir, en los primeros el paciente asevera mucho más que el terapeuta. En cuanto al uso de la Negación, se pudo apreciar un patrón inverso en los Episodios de Cambio comparados con los de Estancamiento. Mientras en los primeros es el terapeuta quien niega con mayor frecuencia, en los segundos es el paciente. Por último, cuando se comparó el uso de Preguntas en los dos tipos de episodio, se pudo apreciar que, siendo los terapeutas los que siempre preguntan más, lo hacen con mayor frecuencia aún en los Episodios de Estancamiento.

Estos hallazgos indican que en los Episodios de Cambio predomina un discurso más propositivo por parte de los terapeutas (con mayor uso de aseveraciones y negaciones) y más receptivo por parte de los pacientes, quienes asienten más. En contraste, en los Episodios de Estancamiento el terapeuta sigue más el discurso del paciente, al menos en la forma verbal básica, Asintiendo más y Negando y Aseverando menos (véase síntesis en Tabla VII).

En cuanto a las intenciones implícitas en la comunicación de cada hablante, también se aprecian importantes diferencias entre los dos tipos de episodio. Si bien la Intención Comunicacional Explorar, vale decir, de solicitar o entregar información, predomina en general por sobre las otras dos intenciones (Resignificar y Sintonizar), este predominio es mayor en los Episodios de Estancamiento que en los de Cambio y es específicamente el paciente quien está predominantemente centrado en un nivel más explorativo, de entregar o solicitar información.

Lo anterior es coherente con lo que muestran los resultados acerca de la Intención Comunicacional Resignificar. En los Episodios de Cambio ambos participantes se comunican con la intención de Resignificar, vale decir, de construir nuevos significados al hablar, mientras que en los de Estancamiento, este esfuerzo está solamente presente en el terapeuta, quien propone nuevos significados, pero sin encontrar eco en el paciente.

Sin embargo, en cuanto a las verbalizaciones que tienen por objetivo mantener la Sintonía entre ambos hablantes, los resultados muestran que, en especial en los Episodios de 
Estancamiento -y a pesar de que el trabajo terapéutico no está siendo productivo en términos de producir cambios de significado-, ambos actores ponen esfuerzo en mantenerse Sintonizados con el otro.

En síntesis, la diferencia fundamental entre Episodios de Cambio y de Estancamiento, en el nivel de las Intenciones Comunicacionales, está en que en los primeros predomina la construcción de nuevos significados, por parte de ambos, quienes los construyen en interacción, mientras en los segundos los terapeutas igualmente intentan buscar nuevos significados, pero sus pacientes mantienen su narrativa, permaneciendo orientados a la entrega de información más que a la construcción de algo nuevo (véase Tabla VII). En ambos tipos de episodio, sin embargo, hay preocupación por mantener la conexión con el otro, a través de verbalizaciones orientadas a la Sintonía. Esto muestra que la comunicación favorecedora de aspectos involucrados en la alianza terapéutica constituye una especie de sustrato que es transversal (Horvath, Del Re, Flückiger \& Symonds, 2011; Krause, Altimir \& Horvath, 2011), y en cierto sentido independiente, de las fluctuaciones en la construcción del cambio terapéutico.

En relación con el uso de Técnicas Comunicacionales, vale decir, la aplicación de ciertos recursos comunicacionales que son visibles tanto en la comunicación cotidiana como en la terapéutica, los resultados mostraron que las de mayor predominio son Argumentación, Confrontación e Interpretación, coincidiendo con los hallazgos de diversos estudios que se han realizado sobre el uso de técnicas terapéuticas (Hill, 2005). Las diferencias en el uso de estos recursos ponen en evidencia la diferencia de roles de los participantes del diálogo terapéutico, ya que en los pacientes predomina la Argumentación, en tanto en los terapeutas, si bien también aparece con una frecuencia relativamente alta, es superada por la Interpretación y la Confrontación, técnicas que los terapeutas utilizan con particular intensidad en Episodios de Estancamiento.

En términos generales, los resultados mostraron un discurso más variado en cuanto al uso de Técnicas en los terapeutas, en comparación con los pacientes, en quienes predominan las verbalizaciones Argumentativas. El hecho de que en los terapeutas se exacerbe el uso de la Confrontación y la Interpretación en Episodios de Estancamiento (Tabla VII) puede ser interpretado en el sentido de un "mayor trabajo" terapéutico, quizás justamente por encontrarse el paciente estancado en sus narrativas.

En el nivel de los contenidos de la comunicación terapéutica, vale decir, si se está hablando de temáticas relativas al Ámbito de la Acción, de las Ideas, o de los Afectos, los resultados mostraron, en primer lugar, el predomino general de las Ideas. En la comparación de ambos participantes, en cuanto a las frecuencias de verbalizaciones correspondientes a los tres Ámbitos, aparecen ciertas diferencias de acuerdo al rol, con un predominio de los contenidos referidos a las Ideas en los terapeutas y de los referidos a la Acción en los pacientes (aunque -como ya se dijera-en ambos predomina el ámbito de las Ideas por sobre los demás). Sin embargo, no es el foco en las Ideas, sino la presencia de los Afectos en el discurso de los hablantes, lo que finalmente distingue Episodios de Cambio y Estancamiento. En los primeros, los pacientes se focalizan más frecuentemente en las emociones que los terapeutas, en tanto en los segundos, los de Estancamiento, ambos se focalizan por igual. En otras palabras, cuando se está produciendo cambio terapéutico, los roles son complementarios, más que en los Episodios de Estancamiento, aportando los pacientes más contenidos afectivos y los terapeutas menos, a favor del ámbito de las Ideas.

Por último, en cuanto al uso de la Referencia en el discurso de los hablantes (acerca de quién están hablando), que es el segundo aspecto de la dimensión de contenidos, los resultados también mostraron distinciones entre Episodios de Cambio y Estancamiento. En términos generales, la Referencia está fuertemente marcada por la complementariedad de los roles en el diálogo terapéutico. Así, mientras en los pacientes predomina la Referencia a sí mismo, en los terapeutas predomina la Referencia al otro presente. Vale decir, ambos están hablando centralmente del paciente y son las alteraciones de este patrón general las que resultan indicativas del tipo de episodio. Así, mientras en los Episodios de Cambio se presenta el patrón esperado, es decir, que el paciente hable de sí mismo y el terapeuta hable del paciente, en los Episodios de Estancamiento ambos presentan un aumento de las verbalizaciones referidas a un tercero y a la relación terapéutica.

Hablar de terceros puede ser interpretado como un desvío del foco óptimo del trabajo terapéutico, y por lo tanto característico de un diálogo que no está aportando al cambio, en tanto la referencia a la relación terapéuti$c a$, probablemente esté al servicio de intentar resolver el Estancamiento, en la línea que se aprecia también en el concepto de resolución de rupturas de la alianza terapéutica (Safran \& Muran, 1996).

En la Tabla VII se presenta una síntesis de los aspectos más característicos de la comunicación de pacientes y terapeutas en los Episodios de Cambio y de Estancamiento, en cada una de las dimensiones de la acción comunicacional estudiadas.

\section{Vínculos con el ejercicio clínico}

Habiendo descrito las diferencias esenciales entre Cambio y Estancamiento desde los resultados del presente estudio, cabría preguntarse: ¿Cómo puede un terapeuta distinguirlos durante la sesión, de modo de favorecer la construcción de Episodios de Cambio e intervenir distinto en los de Estancamiento? A partir de una interpretación de los resultados obtenidos es posible hacer algunos alcances que pueden facilitar la detección de este tipo de episodios durante el ejercicio clínico. 
- En los Episodios de Cambio, aparece un diálogo fructífero entre terapeuta y consultante, donde ambos van construyendo nuevos significados y se logra en el paciente una posición nueva del yo (Aristegui et al, 2009).

- En los Episodios de Estancamiento, en cambio, el paciente muestra un discurso más cerrado, impermeable a la influencia del terapeuta, donde éste no encuentra respuesta a sus intervenciones, dificultándose la generación de un diálogo terapéutico alternativo y por ende la construcción de nuevos significados (Aristegui et al., 2009).

- El terapeuta, en los Episodios de Cambio, interviene de manera directa y asertiva sobre el discurso del paciente, aseverando y negando más, proponiendo verbalizaciones que cuestionan los significados existentes y buscan construir nuevas perspectivas sobre los aspectos que se están trabajando en la terapia. En los Episodios de Cambio aparece un terapeuta que utiliza en forma más flexible una variedad de técnicas comunicacionales, tanto aquellas propias de su enfoque, como otras más genéricas, que también forman parte de las conversaciones cotidianas.

- En cambio, en Episodios de Estancamiento, el terapeuta por una parte exacerba el uso de la técnica (por ejemplo interpretando más), pero por otra, en el nivel de la forma, lo hace con menos asertividad, asintiendo más. Ambas estrategias lo alejan de la posibilidad de construir "momentos de encuentro" (Stern et al., 1998) o de "relación yo-tú" de Buber (1977). Posiblemente como consecuencia de que el cambio no se desarrolla fluidamente, el terapeuta está menos libre para actuar fuera de su rol y se desenvuelve menos espontáneamente.

- En relación con los contenidos del diálogo terapéutico, en los Episodios de Cambio el paciente habla de sí mismo, asumiendo su protagonismo en las dinámicas que le afectan, y el terapeuta aporta en la mantención de este foco.

- En cambio, en los Episodios de Estancamiento ambos actores se desvían parcialmente del foco en el paciente, incluyendo en la conversación más a otras personas (en comparación con los momentos de cambio). Por ejemplo, a veces el paciente habla un largo período acerca de cómo otros afectan su bienestar, sin que la conversación terapéutica logre dirigirse a cómo el mismo participa en sostener esa dinámica.

- Por último, si bien en la conversación terapéutica predominan los contenidos del "mundo de las ideas", es justamente en los Episodios de Cambio, donde el paciente aporta una mayor conexión con los afectos.

\section{Vínculos con la relación terapéutica}

Es relevante señalar que, a pesar de que se aprecian las diferencias señaladas en el comportamiento de paciente y terapeuta según el tipo de episodio observado, hay aspectos de la interacción que se mantienen y que podrían dar luces sobre el trabajo de mantención de la relación terapéutica, que es paralelo y simultáneo al trabajo orientado al cambio en el paciente, y que incluye la adherencia al rol por parte de ambos. Así, aunque se pueda afirmar que en los Episodios de Cambio el terapeuta actúa más espontáneamente, su rol se mantiene. Aunque en estos episodios la relación se vuelve algo más horizontal, se preserva la asimetría, la que se puede observar en que el paciente asevera más, mientras que el terapeuta se dedica más a preguntar; en que el paciente comunica con intención de explorar, mientras que el terapeuta busca principalmente sintonizar y resignificar; en que el paciente se centra más en conductas mientras el terapeuta habla a un nivel más abstracto, generando un espacio de reflexión acerca de los hechos de la vida del paciente; y, finalmente, en que, a pesar de que esporádicamente aparece la auto-revelación como técnica comunicacional del terapeuta, es el paciente quien habla muchísimo más de sí mismo.

Por otra parte, independientemente del tipo de episodio, vale decir, de si el cambio se está construyendo, o bien el diálogo está detenido en narrativas estancadas, tanto el paciente como el terapeuta hacen esfuerzos por preservar la relación, por mantener el entendimiento mutuo, usando un lenguaje que busca la sintonía con el otro. Así, aunque el cambio no se desarrolle por un tiempo, la relación se mantiene como sustrato poderoso que hará posible un nuevo cambio en momentos posteriores.

\section{Limitaciones del estudio y posibilidades futuras}

Finalmente, es pertinente incluir una reflexión sobre algunas limitaciones de este estudio, a fin de visualizar posibles perspectivas para futuras investigaciones. Una limitación conceptual, o epistemológica, deriva de las definiciones mismas de cambio y estancamiento en que se sustenta este trabajo. Por razones más ligadas a las necesidades y restricciones de investigación que a la naturaleza del fenómeno, la definición de cambio y estancamiento utilizada en este trabajo los conceptualiza como algo observable en el paciente, como la construcción de nuevos significados, o la persistencia de patrones disfuncionales típicos. A diferencia de las definiciones utilizadas, tanto el cambio como el estancamiento podrían ser comprendidos como "emergentes relacionales", como aspectos inseparables de la dinámica terapeuta-paciente. Una perspectiva de este tipo, para ser sometida a investigación, debiera probablemente ampliar la unidad de análisis para focalizar en aspectos tales como la regulación mutua entre pacientes y terapeutas, más que en los aportes individuales que cada cual hace al diálogo.

Otra limitación de este estudio deriva de la opción por estudiar aspectos "genéricos" de la comunicación terapéutica, vale decir, transversales a diferentes escuelas y modalidades. Si bien esta búsqueda de aspectos comunes a partir de una realidad heterogénea puede constituir un 
aporte a visiones más integrativas del ejercicio terapéutico, no es menos cierto que vela las diferencias entre los distintos enfoques. En futuros estudios se podría profundizar en la determinación de estas distinciones para profundizar el conocimiento sobre aspectos de la comunicación que son específicos a cada orientación.

Por último, la selección de dos tipos de episodio contrastantes permitió profundizar en aspectos comunicacionales ligados al cambio versus aquellos que aparecen cuando la transformación se estanca, pero omitió el estudio de otros segmentos de la terapia, tales como, por ejemplo, segmentos de ruptura de la relación terapéutica, o segmentos que son preparatorios del cambio sin constituir cambio propiamente tal. Futuros estudios tiene el desafío de profundizar en el análisis de la interacción comunicacional en otros tipos de episodios terapéuticos.

\section{Referencias}

Aristegui, R., Gaete, J., Muñoz, G., Salazar, J., Krause, M., \& Vilches, O. (2009). Diálogos y autorreferencia: Procesos de cambio en psicoterapia desde la perspectiva de los actos de habla. Revista Latinoamericana de Psicología, 41, 277-289.

Arkowitz, H. (2002). Toward an integrative perspective on resistance to change. Psychotherapy in Practice, 58, 219-227.

Bänninger-Huber, E. (1992). Prototypical affective microsequences in psychotherapeutic interaction. Psychotherapy Research, 2, 291-306.

Bänninger-Huber, E., \& Widmer, C. (1999). Affective relationship patterns and psychotherapeutic change. Psychotherapy Research, 9, 74-87.

Bastine, R., Fiedler, P., \& Kommer, D. (1989). Was ist therapeutisch an der Psychotherapie?: Versuch einer Bestandsaufnahme und Systematisierung der Psychotherapeutischen Prozebforschung. Zeitschrift für Klinische Psychologie, 18, 3-22.

Billow, R. M. (2007). On refusal. International Journal of Group Psychotherapy, 57, 419-449.

Brehm, J. W., \& Brehm, S. S. (1981). Psychological reactance: A theory of freedom and control. San Diego, CA: Academic Press.

Breuer, J., \& Freud, S. (1895). Estudios sobre Histeria. El caso de Anna $O$. Obras Completas, Vol II. Buenos Aires: Amorrortu.

Buber, M. (1977). Yo y Tú. Buenos Aires: Nueva Visión.

Elliott, R. (1984). A discovery-oriented approach to significant change events in psychotherapy: Interpersonal process recall and comprehensive process analysis. In L. Rice, \& L. S. Greenberg (Eds.), Patterns of change: Intensive analysis of psychotherapy process (pp. 249-286). New York: Guilford Press.

Elliott, R. (2010). Psychotherapy change process research: Realizing the promise. Psychotherapy Research, 20, 123-135.

Elliott, R., Slatick, E., \& Urman, M. (2001). Qualitative change process research on Psychotherapy: Alternative strategies. En J. Frommer, \& D. L. Rennie (Eds.), Qualitative psychotherapy research: Methods and methodology (pp. 69 -111). Lengerich, Germany: Pabst Science Publishers.

Etchegoyen, H. (1986). Los fundamentos de la técnica psicoanalítica. Buenos Aires: Amorrortu.

Friedlander, M. L. (1982). Counseling discourse as a speech event: Revision and extension of the Hill Counselor Verbal Response Category System. Journal of Counseling Psychology, 29, 425-429.

Gonçalves, M.M., Matos, M., \& Santos, A. (2009). Narrative therapy and the nature of "innovative moments" in the construction of change. Journal of Constructivist Psychology, 22, 1-23.

Herrera, P., Fernández, O., Krause, M., Vilches, O., Valdés, N., \& Dagnino, P. (2009). Revisión teórica y metodológica de las dificultades en
Psicoterapia: Propuesta de un Modelo Ordenador. Terapia Psicológica, $27,169-179$.

Hill, C. (2005). Therapist techniques, client involvement, and the therapeutic relationship: Inextricably intertwined in the therapy process. Psychotherapy: Theory, Research, Practice, Training, 42, 431-442.

Horvath, A. O., Del Re, A. C., Flückiger, C., \& Symonds, B. D. (2011). The alliance. In J. C. Norcross (Ed.), Relationships that work (2ed.). New York, NY: Oxford University Press.

Krause, M. (2005). Psicoterapia y cambio. Una mirada desde la subjetividad. Santiago de Chile: Ediciones Universidad Católica de Chile.

Krause, M. (2011). La Psicoterapia: ¿Oficio sin ciencia y ciencia sin oficio? Revista Colombiana de Psicología, 20, 89-98.

Krause, M., Altimir, C., \& Horvath, A. O. (2011). Deconstructing the therapeutic alliance: Reflections on the underlying dimensions of the concept. Clínica y Salud, 22, 267-283.

Krause, M., De la Parra, G., Arístegui, R., Dagnino, P., Tomicic, A., \& Valdés, N. (2007). The evolution of therapeutic change studied through generic change indicators. Psychotherapy Research, 17, 673-689.

Krause, M., De la Parra, G., Arístegui, R., Tomicic, A., Dagnino, \& P., Echávarri, O. (2006). Indicadores de cambio genéricos en la investigación psicoterapéutica. Revista Latinoamericana de Psicología, 38, 299-325.

Krause, M., Echávarri, O., Ramírez, I., Valdés, N., \& Vilches, O. (Agosto, 2007). Aprender a observar para hacer visible: Cambio y estancamiento en la terapia. Ponencia en el $8^{\circ}$ Congreso Chileno de Psicoterapia: Convergencias y singularidades en psicoterapia. Viña del Mar, Chile.

Martínez, C. (2011). Regulación mutua y dialogicidad en psicoterapia. Saarbrücken, Alemania: Editorial Académica Española.

Matos, M., Santos, A., Goncalves, M., \& Martins, C. (2009). Innovative moments and change in narrative therapy. Psychotherapy Research, $19,68-80$

Reyes, L., Arístegui, R., Krause, M., Strasser, K., Tomicic, A., \& Valdés, N. (2008). Language and Therapeutic Change: A Speech Acts Analysis. Psychotherapy Research 18, 355-362.

Safran, J., \& Muran, J. (2000). Negotiating the Therapeutic Alliance: A Relational Treatment Guide. New York: The Guilford Press.

Searle, J. (1992). Intencionalidad: Un ensayo en la filosofia de la mente. Madrid, España: Tecnos.

Searle, J. (2002). Consciousness and language. Cambridge, UK: Cambridge University Press.

Stern, D., Sander, L., Nahum, J., Harrison, A., Lyons-Ruth, K., \& Morgan, A. (1998). Non-Interpretive mechanisms in Psychoanalytic Therapy. The something more than interpretation. International Journal of Psycho-Analysis, 79, 903-921.

Timulak, L., \& Elliott, R. (2003). Empowerment Events in ProcessExperiential Psychotherapy of Depression: An exploratory qualitative analysis. Psychotherapy Research, 13, 443-460.

Tomicic, A., Martínez, C., Chacón, L.D., \& Guzmán, M. (2011). Patrones de cualidad vocal en psicoterapia: Desarrollo y estudio de confiabilidad de un sistema de codificación. Psykhe, 20, 77-93.

Valdés, N., Krause, M., Vilches, O., Dagnino, P., Echávarri, O., \& Ben-Dov, P. (2005). Proceso de cambio psicoterapéutico: Análisis de episodios relevantes en una terapia grupal con pacientes adictos. Psykhe, 14, 3-18.

Valdés, N. (2010). Análisis de los estilos lingüísticos de paciente y terapeuta durante la conversación terapéutica en episodios de cambio, utilizando el Buscador Lingüístico y Contado de Palabras (LIWC). Revista Subjetividad y Procesos Cognitivos, 14, 314-332.

Valdés, N., Krause, M., \& Álamo, N. (2011). Qué dicen y cómo lo dicen: Análisis de la comunicación verbal de pacientes y terapeutas en episodios de cambio. Revista Argentina de Clínica Psicológica, 20, 15-28.

Valdés, N., Tomicic, A., Pérez, J.C., \& Krause, M. (2010). Sistema de codificación de la actividad terapéutica (SCAT-1.0): Dimensiones y categorías de las acciones comunicacionales de pacientes y psicoterapeutas. Revista Argentina de Clínica Psicológica, 19, 117-130. 


\section{Anexo 1}

\section{Listado de Indicadores de Cambio Genéricos}

\section{Indicadores de Nivel I}

1. Aceptación de la existencia de un problema.

2. Aceptación de los propios límites y reconocimiento de la necesidad de ayuda.

3. Aceptación del terapeuta como un profesional competente.

4. Expresión de esperanza ("remoralización" o expectativa de ser ayudado a superar los problemas).

5. Descongelamiento (o cuestionamiento) de formas de entendimiento, comportamiento y emociones habituales ("fisura"). Puede implicar reconocimiento de problemas antes no vistos, autocrítica $\mathrm{y} / \mathrm{o}$ redefinición de expectativas y metas terapéuticas.

6. Expresión de la necesidad de cambio.

7. Reconocimiento de la propia participación en los "problemas".

\section{Indicadores de Nivel II}

8. Descubrimiento de nuevos aspectos de sí mismo.

9. Manifestación de un comportamiento o de una emoción nueva.

10. Aparición de sentimientos de competencia.

11. Establecimiento de nuevas relaciones entre:

- aspectos propios (creencias, conductas, emociones, etc.)
- aspectos propios y del entorno (personas o sucesos)

- aspectos propios y elementos biográficos

12. Reconceptualización de los propios problemas y/o síntomas.

13. Transformación de valoraciones y emociones en relación a sí mismo u otros. Transformación de la representación de sí mismo y de las propias relaciones con el entorno.

\section{Indicadores de Nivel III}

14. Formación de constructos subjetivos de sí mismos a través de la interconexión de aspectos personales y del entorno, incluyendo problemas y síntomas.

15. Enraizamiento de los constructos subjetivos en la propia biografía.

16. Autonomía en cuanto al manejo propio del contexto de significado psicológico.

17. Reconocimiento de la ayuda recibida.

18. Disminución de la asimetría.

19. Construcción de una Teoría Subjetiva, biográficamente fundada, sobre sí mismo y la relación con el entorno (indicador "global"). 


\section{Anexo 2}

\section{Listado de Temáticas de Estancamiento}
A. Negación de la existencia de un problema o su minimización
B. Negación de la necesidad de ayuda y no aceptación de los propios límites
C. Expresión de desesperanza (desmoralización)
D. No hacerse responsable de los propios actos
E. Aparición de sentimientos de incompetencia
F. Aumento de temor o ambivalencia frente al cambio
G. Atribución a otros de los propios problemas
H. Resistencia a pensar acerca de nuevas posibilidades de sí mismo (conducta, pensamiento o emoción)
I. Cuestionamiento del terapeuta como profesional competente
J. Resistencia al establecimiento de relaciones entre síntomas, emociones y comportamientos
K. Resistencia a la reconceptualización de las definiciones iniciales del problema o síntoma 